\title{
Local Martingale and Pathwise Solutions for an Abstract Fluids Model
}

\author{
Arnaud Debussche ${ }^{\sharp}$, Nathan Glatt-Holtz ${ }^{b}$ and Roger Temam ${ }^{b}$ \\ \#IRMAR and ENS Cachan Bretagne \\ 35170 Bruz, France \\ ${ }^{b}$ The Institute for Scientific Computing and Applied Mathematics \\ Indiana University, Bloomington, IN 47405, USA
}

November 14, 2018

\begin{abstract}
We establish the existence and uniqueness of both local martingale and local pathwise solutions of an abstract nonlinear stochastic evolution system. The primary application of this abstract framework is to infer the local existence of strong, pathwise solutions to the $3 D$ primitive equations of the oceans and atmosphere forced by a nonlinear multiplicative white noise. Instead of developing our results specifically for the $3 D$ primitive equations we choose to develop them in a slightly abstract framework which covers many related forms of these equations (atmosphere, oceans, coupled atmosphere-ocean, on the sphere, on the $\beta$-plane approximation etc and the incompressible Navier-Stokes equations). In applications, all of the details are given for the $\beta$-plane approximation of the oceans equations.
\end{abstract}

\section{Introduction}

In this work we develop a local existence theory for a class of abstract stochastic evolution systems of the form:

$$
d U+(A U+B(U, U)+F(U)) d t=\sigma(U) d W, \quad U(0)=U_{0},
$$

which includes the primitive equations of the ocean as explained below. We choose to develop our setting in a somewhat abstract framework so that our results may cover some closely related equations such as the primitive equations of the atmosphere or the coupled atmospheric/oceanic system, equations with chemistry, equations on the sphere or $\beta$-plane approximations, etc. We do not expand further on these latter applications in this article in order to avoid excessive developments. The stochastic primitive equations of the ocean have been previously studied in $[26,22,24,15,27]$ but none of these works address the full 3 -d system in the context of a nonlinear multiplicative noise.

The deterministic primitive equations are widely seen as a fundamental model for large scale oceanic and atmospheric systems. For the oceans they are derived from the fully compressible Navier-Stokes equations combined with the Boussinesq and hydrostatic approximations. See e.g. [39] for further physical background. Given the growing importance of probabilistic methods in sub-grid scale parameterization, geophysicists currently devote significant attention to stochastic forms of the equations of geophysical fluid dynamics; see e.g. $[14,40,41,44,32,34,4,50]$. From the mathematical point of view the stochastic primitive equations have been considered for a two dimensional version of the equations in $[26,15,25]$. In three space dimensions [27] has addressed the case of an additive noise. In this situation the outcome $\omega$ (in the underlying probability space $\Omega$ ) may be treated as a parameter in the problem. Furthermore the methods in [27] do not allow for physically realistic boundary conditions which we are able to treat here with our methods. 
Let us recall at this point that the theory of the related stochastic Navier-Stokes equations have undergone substantial developments; see e.g. $[3,48,9,8,17,12,2,5,6,19,10,36,37,45,18,23]$. However to emphasize the notable differences between the stochastic Navier-Stokes Equations and the primitive equations we recall that the deterministic Primitive equations are known to be well posed in space dimension three (see [7, 30, 31]). This result is not known for the Navier-Stokes Equations and is the object of the famous Clay problem. On the other hand the primitive equations are technically more involved than the NavierStokes equations. For further background concerning the mathematical theory for the deterministic primitive equations see e.g. the review article [42] and the references therein. The stochastic primitive equations that we consider in this work are described in detail in Section 6 below.

In the theory of stochastic evolution equations two notions of solutions are typically considered namely pathwise (or strong) solutions and martingale (or weak) solutions. In the former notion the driving noise is fixed in advance while in the later case these underlying stochastic elements enter as an unknown in the problem. In this work we will consider both notions for (1.1) and illuminate the relationship between these two types of solutions. The classical Yamada-Watanabe theorem from finite dimensional stochastic analysis says that pathwise solutions exist whenever martingale solutions may be found and pathwise uniqueness of solutions holds (see e.g. [43]). Similar results have later been established along more elementary lines, in [28], using a simple characterization of convergence in probability (see Proposition 2.2 below). This characterization may also be employed in the infinite dimensional context and is used below to pass from the case of martingale to pathwise solutions. In any case to the best of our knowledge, no one has previously established such a 'Yamada-Watanabe' type result for the Primitive Equations (or for that matter for the $3 D$ Navier-Stokes equations).

The exposition is organized as follows. In Section 2 we make precise the set-up of the abstract problem (1.1) and briefly recall some relevant mathematical preliminaries from probability theory and functional analysis. A Galerkin scheme for (1.1) is considered in Section 3. By making use of an appropriate cutoff function in the formulation of the equations we are able to establish uniform a-priori estimates for the corresponding sequence of approximate solutions. In Section 4 we outline the compactness arguments that lead to the local existence of martingale solutions. We turn then to pathwise solutions in Section 5. Here the first step is to establish conditions for pathwise uniqueness. We then revisit the compactness methods described in the previous section now making use of the additional criteria for convergence in probability. In Section 6 we apply the abstract results to the stochastic primitive equations. In the final Sections, 7, 8, we provide the technical details of the passage to the limit from compactness of Galerkin approximations established earlier in Sections 4, 5 .

\section{Mathematical Framework}

In this section we set up the abstract system (1.1), making precise the conditions on each of the terms and reviewing the notions of both martingale and pathwise solutions. We also recall various results from abstract probability theory and functional analysis which play a fundamental role in the analysis.

\subsection{Abstract Spaces and Operators}

We begin by fixing a pair of separable Hilbert spaces $H \supset V$, and assume that the embedding is dense and compact. We may thus define the Gelfand inclusions $V \subset H \subset V^{\prime}$, where $V^{\prime}$ is the dual of $V$, relative to $H$. We denote by $(\cdot, \cdot),|\cdot|,((\cdot, \cdot))$ and $\|\cdot\|$ the norms and inner products of $H$, and $V$ respectively. The duality product between $V^{\prime}$ and $V$ is written $\langle\cdot, \cdot\rangle$.

\subsubsection{The Principal Linear Operator}

We now give the precise assumptions on each of the terms appearing in (1.1). They are of course designed to include the case of the primitive equations of the ocean, (6.1)- (6.5) as we explain below in Section 6. We begin with the linear term supposing that $A: D(A) \subset H \rightarrow H$ is an unbounded, densely defined, bijective, 
operator such that $\left(A U, U^{\sharp}\right)=\left(\left(U, U^{\sharp}\right)\right)$ for all $U, U^{\sharp} \in D(A)$. As such we see that $A$ is symmetric and may be understood as bounded operator from $V$ to $V^{\prime}$ with the duality product given by

$$
\left\langle A U, U^{\sharp}\right\rangle=\left(\left(U, U^{\sharp}\right)\right), \quad \text { for all } U \in V \text {. }
$$

We further see that $A^{-1}$ is continuous as a map from $H$ into $V$. Since by assumption $V \subset \subset H$, it follows that $A^{-1}$ is compact on $H$. We may also deduce from the given assumptions on $A$ that $A^{-1}$ is symmetric. Applying the classical theory for symmetric compact operators we infer the existence of a complete orthonormal basis $\left\{\Phi_{k}\right\}_{k \geq 0}$ for $H$ of eigenfunctions of $A$ so that the associated sequence of eigenvalues $\left\{\lambda_{k}\right\}_{k \geq 0}$ form an increasing unbounded sequence. For the Galerkin scheme below we introduce the finite dimensional spaces

$$
H_{n}=\operatorname{span}\left\{\Phi_{1}, \ldots, \Phi_{n}\right\}
$$

and let $P_{n}, Q_{n}=I-P_{n}$ be the projection operators onto $H_{n}$ and its orthogonal complement.

Using the basis $\left\{\Phi_{k}\right\}$ we may also define the fractional powers of $A$ which are also relevant to the analysis. Given $\alpha>0$, take

$$
D\left(A^{\alpha}\right)=\left\{U \in H: \sum_{k} \lambda_{k}^{2 \alpha}\left|U_{k}\right|^{2}<\infty\right\}
$$

where $U_{k}=\left(U, \Phi_{k}\right)$. On this set we may define $A^{\alpha}$ according to

$$
A^{\alpha} U=\sum_{k} \lambda_{k}^{\alpha} U_{k} \Phi_{k}, \quad \text { for } U=\sum_{k} U_{k} \Phi_{k} .
$$

Accordingly we equip $D\left(A^{\alpha}\right)$ with the Hilbertian norm

$$
|U|_{\alpha}:=\left|A^{\alpha} U\right|=\left(\sum_{k} \lambda_{k}^{2 \alpha}\left|U_{k}\right|^{2}\right)^{1 / 2} .
$$

Classically we have the following generalized Poincaré and inverse Poincaré inequalities:

$$
\left|P_{n} U\right|_{\alpha_{2}} \leq \lambda_{n}^{\alpha_{2}-\alpha_{1}}\left|P_{n} U\right|_{\alpha_{1}}, \quad\left|Q_{n} U\right|_{\alpha_{1}} \leq \frac{1}{\lambda_{n}^{\alpha_{2}-\alpha_{1}}}\left|Q_{n} U\right|_{\alpha_{2}},
$$

valid for any $\alpha_{1}<\alpha_{2}$.

Note that as in [46] one may verify that $D\left(A^{\beta}\right) \subset D\left(A^{\alpha}\right)$ is a compact embedding whenever $\beta>\alpha$. Using (2.1), one may readily verify that $D\left(A^{1 / 2}\right)=V$ and that $\|U\|=|U|_{1 / 2}$ for all $U \in V$. Thus, it is clear that, in particular, the embedding $D(A) \subset V$ is compact.

\subsubsection{The Nonlinear Terms}

We turn next to $B$ which we assume to be a bilinear form mapping $V \times D(A)$ continuously to $V^{\prime}$ and $D(A) \times D(A)$ continuously to $H$. Furthermore we assume the following properties for $B$ :

$$
\begin{gathered}
\left\langle B\left(U, U^{\sharp}\right), U^{\sharp}\right\rangle=0 \quad \text { for all } U \in V, U^{\sharp} \in D(A), \\
\left|\left\langle B\left(U, U^{\sharp}\right), U^{\mathrm{b}}\right\rangle\right| \leq c_{0}\|U\|\left|A U^{\sharp}\right|\left\|U^{\mathrm{b}}\right\| \quad \text { for all } U, U^{\mathrm{b}} \in V, U^{\sharp} \in D(A), \\
\left|\left\langle B\left(U, U^{\sharp}\right), U^{\mathrm{b}}\right\rangle\right| \leq c_{0}\|U\|^{1 / 2}|A U|^{1 / 2}\left\|U^{\sharp}\right\|^{1 / 2}\left|A U^{\sharp}\right|^{1 / 2}\left|U^{\mathrm{b}}\right| \quad \text { for all } U, U^{\sharp} \in D(A), U^{\mathrm{b}} \in H .
\end{gathered}
$$

Note that, for brevity of notation, we will sometimes write $B(U)$ for $B(U, U)$.

We next describe the conditions imposed for $F$ and $\sigma$. To this end we introduce some further notations. Given any pair of Banach spaces $\mathcal{X}$ and $\mathcal{Y}$ we denote by $B n d_{u}(\mathcal{X}, \mathcal{Y})$, the collection of all continuous mappings

$$
\Psi:[0, \infty) \times \mathcal{X} \rightarrow \mathcal{Y}
$$


so that

$$
\|\Psi(x, t)\|_{\mathcal{Y}} \leq c\left(1+\|x\|_{\mathcal{X}}\right), \quad x \in \mathcal{X}, t \geq 0
$$

where the numerical constant $c$ may be chosen independently of $t$. If, in addition,

$$
\|\Psi(x, t)-\Psi(y, t)\|_{\mathcal{Y}} \leq c\|x-y\|_{\mathcal{X}}, \quad x, y \in \mathcal{X}, t \geq 0
$$

we say $\Psi$ is in $\operatorname{Lip}_{u}(\mathcal{X}, \mathcal{Y})$.

For $F$ we assume that

$$
F:[0, \infty) \times V \rightarrow H
$$

In Section 4 we assume that

$$
F \in \operatorname{Bnd}_{u}(V, H)
$$

Further on in Section 5

$$
F \in \operatorname{Lip}_{u}(V, H) \text {. }
$$

Similar conditions are also imposed on $\sigma$. We shall assume throughout this work that

$$
\sigma:[0, \infty) \times H \rightarrow L_{2}(\mathfrak{U}, H) .
$$

Here $\mathfrak{U}$ is an auxiliary Hilbert space and $L_{2}(\mathfrak{U}, H)$ is the collection of Hilbert-Schmidt operators between $\mathfrak{U}$ and $H$. See Section 2.2 for further remarks. For the case of martingale solutions considered in Section 4 , we assume that

$$
\sigma \in \operatorname{Bnd}_{u}\left(H, L_{2}(\mathfrak{U}, H)\right) \cap \operatorname{Bnd}_{u}\left(V, L_{2}(\mathfrak{U}, V)\right) \cap B n d_{u}\left(D(A), L_{2}(\mathfrak{U}, D(A))\right) .
$$

On the other hand for pathwise solutions, Section 5 , we posit

$$
\sigma \in \operatorname{Lip}_{u}\left(H, L_{2}(\mathfrak{U}, H)\right) \cap \operatorname{Lip}_{u}\left(V, L_{2}(\mathfrak{U}, V)\right) \cap \operatorname{Lip}_{u}\left(D(A), L_{2}(\mathfrak{U}, D(A))\right) .
$$

\subsection{The Stochastic Framework}

In order to define the remaining terms in (1.1), that is $\sigma(U) d W$ we must recall some basic notions and notations from stochastic analysis. For more theoretical background on the general theory of stochastic evolution systems we mention the classical book [11] or the more recent treatment in [43].

To begin we fix a stochastic basis $\mathcal{S}:=\left(\Omega, \mathcal{F},\left\{\mathcal{F}_{t}\right\}_{t \geq 0}, \mathbb{P},\left\{W^{k}\right\}_{k \geq 1}\right)$, that is a filtered probability space with $\left\{W^{k}\right\}_{k \geq 1}$ a sequence of independent standard 1-d Brownian motions relative to $\mathcal{F}_{t}$. In order to avoid unnecessary complications below we may assume that $\mathcal{F}_{t}$ is complete and right continuous (see [11]). Fix a separable Hilbert space $\mathfrak{U}$ with an associated orthonormal basis $\left\{e_{k}\right\}_{k \geq 1}$. We may formally define $W$ by taking $W=\sum_{k} W_{k} e_{k}$. As such $W$ is a 'cylindrical Brownian' motion evolving over $\mathfrak{U}$.

We next recall some basic definitions and properties of spaces of Hilbert-Schmidt operators. For this purpose we suppose that $X$ and $\tilde{X}$ are any pair of separable Hilbert spaces with the associated norms and inner products given by $|\cdot|_{X},|\cdot|_{\tilde{X}}$ and $\langle\cdot, \cdot\rangle_{X}\langle\cdot, \cdot\rangle_{\tilde{X}}$, respectively. We denote by

$$
L_{2}(\mathfrak{U}, X)=\left\{R \in \mathcal{L}(\mathfrak{U}, X): \sum_{k}\left|R e_{k}\right|_{X}^{2}<\infty\right\},
$$

the collection of Hilbert-Schmidt operators from $\mathfrak{U}$ to $X$. By endowing this collection with the inner product $\langle R, S\rangle_{L_{2}(\mathfrak{U}, X)}=\sum_{k}\left\langle R e_{k}, S e_{k}\right\rangle_{X}$, we may consider $L_{2}(\mathfrak{U}, X)$ as itself being a Hilbert space. Note that when $R \in L_{2}(\mathfrak{U}, X)$ we shall often denote $R_{k}=R e_{k}$ and we may therefore associate $R$ with the sequence $\left\{R_{k}\right\}_{k \geq 1}$. One may readily show that if $R^{(1)} \in L_{2}(\mathfrak{U}, X)$ and $R^{(2)} \in L(X, \tilde{X})$ then indeed $R^{(2)} R^{(1)} \in L_{2}(\mathfrak{U}, \tilde{X})$.

We also define the auxilary space $\mathfrak{U}_{0} \supset \mathfrak{U}$ via

$$
\mathfrak{U}_{0}:=\left\{v=\sum_{k \geq 0} \alpha_{k} e_{k}: \sum_{k} \alpha_{k}^{2} k^{2}<\infty\right\},
$$


endowed with the norm $|v|_{\mathfrak{U}_{0}}^{2}:=\sum_{k} \frac{\alpha_{k}^{2}}{k^{2}}, \quad v=\sum_{k} \alpha_{k} e_{k}$. Note that the embedding of $\mathfrak{U} \subset \mathfrak{U}_{0}$ is HilbertSchmidt. Moreover, using standard Martingale arguments with the fact that each $W_{k}$ is almost surely continuous (see [11]) we have that, for almost every $\omega \in \Omega, W(\omega) \in C\left([0, T], \mathfrak{U}_{0}\right)$.

Given an $X$-valued predictable ${ }^{1}$ process $G \in L^{2}\left(\Omega ; L_{l o c}^{2}\left([0, \infty), L_{2}(\mathfrak{U}, X)\right)\right)$ one may define the (Itō) stochastic integral

$$
M_{t}:=\int_{0}^{t} G d W=\sum_{k} \int_{0}^{t} G_{k} d W_{k}
$$

as an element in $\mathcal{M}_{X}^{2}$, that is the space of all $X$-valued square integrable martingales (see [43, Section 2.2, 2.3]). As such $\left\{M_{t}\right\}_{t \geq 0}$ has many desirable properties. Most notably for the analysis here, the BurkholderDavis-Gundy inequality holds which in the present context takes the form,

$$
\mathbb{E}\left(\sup _{t \in[0, T]}\left|\int_{0}^{t} G d W\right|_{X}^{r}\right) \leq c \mathbb{E}\left(\int_{0}^{T}|G|_{L_{2}(\mathfrak{U}, X)}^{2} d t\right)^{r / 2},
$$

valid for any $r \geq 1$. Here $c$ is an absolute constant depending only on $r$. We shall also make use of a variation of this inequality, established in [17] which applies to fractional derivatives of $M_{t}$. For $p \geq 2$ and $\alpha \in[0,1 / 2)$ we have

$$
\mathbb{E}\left(\left|\int_{0}^{t} G d W\right|_{W^{\alpha, p}([0, T] ; X)}^{p}\right) \leq c \mathbb{E}\left(\int_{0}^{T}|G|_{L_{2}(\mathfrak{U}, X)}^{p} d t\right),
$$

which holds for all $X$-valued predictable $G \in L^{p}\left(\Omega ; L_{l o c}^{p}\left([0, \infty), L_{2}(\mathfrak{U}, X)\right)\right)$. For the convenience of the reader, we shall recall the definition of the spaces $W^{\alpha, p}([0, T], X)$ in Section 2.4 below.

Remark 2.1. Under the assumptions, (2.10), (2.11), on $\sigma$, the stochastic integral $t \mapsto \int_{0}^{t} \sigma(U) d W$ may be shown to be well defined (in the Ito sense), taking values in $H$ whenever $U \in L^{2}\left(\Omega, L_{\text {loc }}^{2}([0, \infty) ; H)\right.$ ) and is predictable. Such terms may be seen to cover a wide class of examples, including but not limited to the classical cases of additive and linear multiplicative noise, projections of the solution in any direction, and directional forcings of Lipschitz functionals of the solution. See e.g. [23] for further details.

In Section 8 we establish the following convergence theorem for stochastic integrals. This result will be used below to facilitate the passage to the limit in the Galerkin scheme. The statement and proof generalizes ideas found in [1].

Lemma 2.1. Let $(\Omega, \mathcal{F}, \mathbb{P})$ be a fixed probability space, $X$ a separable Hilbert space. Consider a sequence of stochastic bases $\mathcal{S}_{n}=\left(\Omega, \mathcal{F},\left\{\mathcal{F}_{t}^{n}\right\}_{t>0}, \mathbb{P}, W^{n}\right)$, that is a sequence so that each $W^{n}$ is cylindrical Brownian motion (over $\mathfrak{U}$ ) with respect to $\mathcal{F}_{t}^{n}$. Assume that $\left\{G^{n}\right\}_{n \geq 1}$ are a collection of $X$-valued $\mathcal{F}_{t}^{n}$ predictable processes such that $G^{n} \in L^{2}\left([0, T], L_{2}(\mathfrak{U}, X)\right)$ a.s. Finally consider $\mathcal{S}=\left(\Omega, \mathcal{F},\left\{\mathcal{F}_{t}\right\}_{t \geq 0}, \mathbb{P}, W\right)$ and $G \in$ $L^{2}\left([0, T], L_{2}(\mathfrak{U}, X)\right)$, which is $\mathcal{F}_{t}$ predictable. If

$$
\begin{gathered}
W^{n} \rightarrow W \quad \text { in probability in } C\left([0, T], \mathfrak{U}_{0}\right), \\
G^{n} \rightarrow G \quad \text { in probability in } L^{2}\left([0, T] ; L_{2}(\mathfrak{U}, X)\right),
\end{gathered}
$$

then

$$
\int_{0}^{t} G^{n} d W^{n} \rightarrow \int_{0}^{t} G d W \quad \text { in probability in } L^{2}([0, T] ; X) .
$$

\footnotetext{
${ }^{1}$ For a given stochastic basis $\mathcal{S}$, let $\Phi=\Omega \times[0, \infty)$ and take $\mathcal{G}$ to be the $\sigma$-algebra generated by sets of the form$$
(s, t] \times F, \quad 0 \leq s<t<\infty, F \in \mathcal{F}_{s} ; \quad\{0\} \times F, \quad F \in \mathcal{F}_{0} .
$$

Recall that an $X$ valued process $U$ is called predictable (with respect to the stochastic basis $\mathcal{S}$ ) if it is measurable from $(\Phi, \mathcal{G}$ ) into $(X, \mathcal{B}(X)), \mathcal{B}(X)$ being the family of Borel sets of $X$.
} 
Finally we describe the assumptions for the initial condition $U_{0}$ which may be random in general. In Section 4, where we consider the case of Martingale solutions, since the stochastic basis is an unknown of the problem we are only able to specify $U_{0}$ as an initial probability measure $\mu_{0}$ on $V$ such that:

$$
\int_{V}\|U\|^{q} d \mu_{0}(U)<\infty
$$

Here $q \geq 2$ will be specified below, see Theorem 2.1 as well as Lemma 3.1. On the other hand for pathwise solutions where the stochastic basis $\mathcal{S}$ is fixed we assume that relative to this basis $U_{0}$ is a $V$ valued random variable such that

$$
U_{0} \in L^{2}(\Omega ; V) \text { and is } \mathcal{F}_{0} \text { measurable. }
$$

\subsection{Definition of Solutions}

We next give the definitions of local and global solutions of (1.1) for both Martingale and Pathwise Solutions.

Definition 2.1 (Local and Global Martingale Solutions). Suppose $\mu_{0}$ is probability measure on $V$ satisfying (2.16) with $q \geq 8$ and assume that (2.7) and (2.10) hold for $F$ and $\sigma$ respectively.

(i) A triple $(\mathcal{S}, U, \tau)$ is a local Martingale solution if $\mathcal{S}=\left(\Omega, \mathcal{F},\left\{\mathcal{F}_{t}\right\}_{t \geq 0}, \mathbb{P}, W\right)$ is a stochastic basis, $\tau$ is stopping time relative to $\mathcal{F}_{t}$ and $U(\cdot)=U(\cdot \wedge \tau): \Omega \times[0, \infty) \rightarrow V$ is an $\mathcal{F}_{t}$ adapted process such that:

$$
\begin{array}{r}
U(\cdot \wedge \tau) \in L^{2}(\Omega ; C([0, \infty) ; V)), \\
U \mathbb{1}_{t \leq \tau} \in L^{2}\left(\Omega ; L_{l o c}^{2}([0, \infty) ; D(A))\right) ;
\end{array}
$$

the law of $U(0)$ is $\mu_{0}$ i.e. $\mu_{0}(E)=\mathbb{P}(U(0) \in E)$, for all Borel subsets $E$ of $V$, and $U$ satisfies for every $t \geq 0$

$$
U(t \wedge \tau)+\int_{0}^{t \wedge \tau}(A U+B(U)+F(U)) d s=U(0)+\int_{0}^{t \wedge \tau} \sigma(U) d W
$$

with the equality understood in $H$.

(ii) We say that the (Martingale) solution $(\mathcal{S}, U, \tau)$ is global if $\tau=\infty, \Omega$-a.s.

We next define pathwise solutions of (1.1) where the stochastic basis is fixed in advance.

Definition 2.2 (Local, Maximal and Global Pathwise Solutions). Let $\mathcal{S}=\left(\Omega, \mathcal{F},\left\{\mathcal{F}_{t}\right\}_{t \geq 0}, \mathbb{P}, W\right)$ be a fixed stochastic basis and suppose that $U_{0}$ is an $V$ valued random variable (relative to $\mathcal{S}$ ) satisfying (2.17). Assume that $F$ satisfies (2.8) and that (2.11) holds for $\sigma$.

(i) A pair $(U, \tau)$ is a a local pathwise solution of (1.1) if $\tau$ is a strictly positive stopping time and $U(\cdot \wedge \tau)$ is an $\mathcal{F}_{t}$-adapted process in $V$ so that (relative to the fixed basis $\mathcal{S}$ ) (2.18), (2.19) hold.

(ii) Pathwise solutions of (1.1) are said to be (pathwise) unique up to a stopping time $\tau>0$ if given any pair of pathwise solutions $\left(U^{1}, \tau\right)$ and $\left(U^{2}, \tau\right)$ which coincide at $t=0$ on a subset $\tilde{\Omega}$ of $\Omega, \tilde{\Omega}=\left\{U^{1}(0)=\right.$ $\left.U^{2}(0)\right\}$, then

$$
\mathbb{P}\left(\mathbb{1}_{\tilde{\Omega}}\left(U^{1}(t \wedge \tau)-U^{2}(t \wedge \tau)\right)=0 ; \forall t \geq 0\right)=1
$$

(iii) Suppose that $\left\{\tau_{n}\right\}_{n \geq 1}$ is a strictly increasing sequence of stopping times converging to a (possibly infinite) stopping time $\xi$ and assume that $U$ is a predictable process in $H$. We say that the triple $\left(U, \xi,\left\{\tau_{n}\right\}_{n \geq 1}\right)$ is a maximal strong solution if $\left(U, \tau_{n}\right)$ is a local strong solution for each $n$ and

$$
\sup _{t \in[0, \xi]}\|U\|^{2}+\int_{0}^{\xi}|A U|^{2} d s=\infty
$$


almost surely on the set $\{\xi<\infty\}$. If, moreover

$$
\sup _{t \in\left[0, \tau_{n}\right]}\|U\|^{2}+\int_{0}^{\tau_{n}}|A U|^{2} d s=n,
$$

for almost every $\omega \in\{\xi<\infty\}$ then the sequence $\tau_{n}$ is said to announce any finite time blow up.

(iii) If $(U, \xi)$ is a maximal strong solution and $\xi=\infty$ a.s. then we say that the solution is global.

We may now state precisely the main results in the work:

Theorem 2.1.

(i) Suppose that $\mu_{0}$ satisfies (2.16), for $q \geq 8$ and that $F$ and $\sigma$ maintain (2.7), (2.10) respectively. Then there exists a local Martingale solution $(\mathcal{S}, U, \tau)$ of (1.1).

(ii) Assume that, relatively to a fixed stochastic basis $\mathcal{S}, U_{0}$ satisfies (2.17) and that $F$ and $\sigma$ fulfill (2.8) and (2.11). Then there exists a unique, maximal pathwise solution, $\left(U, \xi,\left\{\tau_{n}\right\}_{n \geq 1}\right)$, of (1.1).

The compactness arguments leading to Theorem 2.1 are carried out in Sections 4 and 5 for (i) and (ii) respectively. We provide the details of the passage to the limit needed for both items in Section 7 .

Remark 2.2. (i) We note that, as we are working at the intersection of two fields, the terminology may cause some confusion. In the literature for stochastic differential equations the term "weak solution" is sometimes used synonymously with the term "martingale solution" while the designation "strong solution" may be used for a "pathwise solution". See the introductory text of Øksendal [38] for example. The former terminologies are avoided here because it is confusing in the context of partial differential equations. Indeed, from the PDE point of view, strong solutions are solutions which are uniformly bounded in $H^{1}$, while weak solutions are those which are merely bounded in $L^{2}$. In this work we are therefore considering both weak and strong solutions in probabilistic sense. From the PDE point of view we may say that we are considering strong type solutions since, in the applications considered here $V$ is taken to be an appropriate subspace of $H^{1}$ that incorporates the boundary conditions, etc., for (6.1).

(ii) The notion of global existence, both for the Martingale and the Pathwise contexts, are included here for the sake of completeness. Of course, the passage from the maximal to the global existence of pathwise solutions is a significant further step in the analysis and requires further structure for (1.1). We refer the reader to [23] and [24] where this is done for the 2D Navier-Stokes Equations and the 2D Primitive Equations respectively. Current work, making use of the main result herein, treats the global existence of solutions for the stochastic 3D Primitive Equations [13].

(iii) In Section 5, 7 we consider both Martingale and Pathwise solutions of the modified system

$$
d U+\left(A U+\theta\left(\left\|U-U_{*}\right\|\right) B(U)+F(U)\right) d t=\sigma(U) d W, \quad U(0)=U_{0},
$$

where

$$
\frac{d}{d t} U_{*}+A U_{*}=0, \quad U(0)=U_{0}
$$

and $\theta$ is a smooth cut-off function as defined below in (3.1). The notions of solutions for (2.22) are, with trivial modifications, identical to Definitions 2.1, 2.2 given for (1.1) above.

\subsection{Compact Embedding Theorems}

We shall make use of two compact embedding results taken from [17] which we restate here. See also related results in [46]. To this end we first recall some spaces of fractional (in time) derivative. Such spaces are natural since we do not expect solutions of stochastic evolution systems to be differentiable in time but merely Holder continuous of order strictly less than $1 / 2$. 
Let $X$ be a separtable Hilbert space and denote the associated norm by $|\cdot|_{X}$. For fixed $p>1$ and $\alpha \in(0,1)$ we define

$$
W^{\alpha, p}([0, T] ; X):=\left\{U \in L^{p}([0, T] ; X) ; \int_{0}^{T} \int_{0}^{T} \frac{\left|U\left(t^{\prime}\right)-U\left(t^{\prime \prime}\right)\right|_{X}^{p}}{\left|t^{\prime}-t^{\prime \prime}\right|^{1+\alpha p}} d t^{\prime} d t^{\prime \prime}<\infty\right\} .
$$

We endow this space with the norm

$$
|U|_{W^{\alpha, p}([0, T] ; X)}^{p}:=\int_{0}^{T}\left|U\left(t^{\prime}\right)\right|_{X}^{p} d t^{\prime}+\int_{0}^{T} \int_{0}^{T} \frac{\left|U\left(t^{\prime}\right)-U\left(t^{\prime \prime}\right)\right|_{X}^{p}}{\left|t^{\prime}-t^{\prime \prime}\right|^{1+\alpha p}} d t^{\prime} d t^{\prime \prime} .
$$

For the case when $\alpha=1$ we take $W^{1, p}([0, T] ; X):=\left\{U \in L^{p}([0, T] ; X) ; \frac{d U}{d t} \in L^{p}([0, T] ; X)\right\}$, to be the classical Sobolev space with its usual norm

$$
|U|_{W^{1, p}([0, T] ; X)}^{p}:=\int_{0}^{T}\left|U\left(t^{\prime}\right)\right|_{X}^{p}+\left|\frac{d U}{d t}\left(t^{\prime}\right)\right|_{X}^{p} d t^{\prime} .
$$

Note that for $\alpha \in(0,1), W^{1, p}([0, T] ; X) \subset W^{\alpha, p}([0, t] ; X)$ and $|U|_{W^{\alpha, p}([0, T] ; X)} \leq C \mid U_{W^{1, p}([0, T] ; X)}$. With these preliminaries in hand we may now state the compact embeddings needed below (see [17])

\section{Lemma 2.2.}

(i) Suppose that $X_{2} \supset X_{0} \supset X_{1}$ are Banach spaces with $X_{2}$ and $X_{1}$ reflexive, and the embedding of $X_{1}$ into $X_{0}$ compact. Then for any $1<p<\infty$ and $0<\alpha<1$, the embedding:

$$
L^{p}\left([0, T] ; X_{1}\right) \cap W^{\alpha, p}\left([0, T] ; X_{2}\right) \subset \subset L^{p}\left([0, T] ; X_{0}\right)
$$

is compact.

(ii) Suppose that $Y_{0} \supset Y$ are Banach spaces with $Y$ compactly embedded in $Y_{0}$. Let $\alpha \in(0,1]$ and $p \in(1, \infty)$ be such that $\alpha p>1$ then

$$
W^{\alpha, p}([0, T] ; Y) \subset \subset C\left([0, T], Y_{0}\right)
$$

and the embedding is compact.

\subsection{Some Tools From Abstract Probability Theory}

We next review some classical convergence results for probability measures defined on separable metric spaces. In conjuction with the embeddings given in Section 2.4, these results provide some powerful means to address the difficulty of establishing compactness for the collection of Galerkin approximations associated to $(1.1)$.

Let $(X, d)$ be a complete separable metric space and take $\mathcal{B}(X)$ to be the associated borel $\sigma$-algebra. Also, we define $C_{b}(X)$ to be the collection of all real valued continuous bounded functions on $X$ and take $\operatorname{Pr}(X)$ to be the set of all probability measures on $(X, \mathcal{B}(X))$. Recall that a collection $\Lambda \subset \operatorname{Pr}(X)$ is said to be tight if, for every $\epsilon>0$, there exists a compact set $K_{\epsilon} \subset X$ such that:

$$
\mu\left(K_{\epsilon}\right) \geq 1-\epsilon \quad \text { for all } \mu \in \Lambda \text {. }
$$

On the other hand a sequence $\left\{\mu_{n}\right\}_{n \geq 0} \subset \operatorname{Pr}(X)$ is said to converge weakly to an probability measure $\mu$ if

$$
\int f d \mu_{n} \rightarrow \int f d \mu
$$

over all $f \in C_{b}(X)$. We say that a set $\Lambda \subset \operatorname{Pr}(X)$ is weakly compact if every sequence $\left\{\mu_{n}\right\} \subset \Lambda$ possesses a weakly convergent subsequence.

Proofs of the following classical results may be found in e.g. [11]. 
Proposition 2.1. (i) A collection $\Lambda \subset \operatorname{Pr}(X)$ is weakly compact if and only if it is tight.

(ii) Suppose that a sequence $\left\{\mu_{n}\right\}_{n \geq 1}$ converges weakly to a measure $\mu$. Then there exists a probability space $(\tilde{\Omega}, \tilde{\mathcal{F}}, \tilde{\mathbb{P}})$ and a sequence of $X$ valued random variables $\left\{\tilde{Y}_{n}\right\}_{n \geq 0}$ (relative to this space) such that $\tilde{Y}_{n}$ converges almost surely to the random variable $\tilde{Y}$ and such that the laws of $\tilde{Y}_{n}$ and $\tilde{Y}$ are $\mu_{n}$ and $\mu$, i.e. $\mu_{n}(E)=\mathbb{P}\left(Y_{n} \in E\right), \mu(E)=\mathbb{P}(Y \in E)$, for all $E \in \mathcal{B}(X)$.

Finally we come to an elementary but powerful characterization of convergence in probability introduced in [28]. Suppose that $\left\{Y_{n}\right\}_{n \geq 0}$ is a sequence of $X$-valued random variables on a probability space $(\Omega, \mathcal{F}, \mathbb{P})$. Let $\left\{\mu_{n, m}\right\}_{n, m \geq 1}$ be the collection of joint laws of $\left\{Y_{n}\right\}_{n \geq 1}$, that is

$$
\mu_{n, m}(E):=\mathbb{P}\left(\left(Y_{n}, Y_{m}\right) \in E\right), \quad E \in \mathcal{B}(X \times X) .
$$

The result from $[28]$ is the following:

Proposition 2.2. A sequence of $X$ valued random variables $\left\{Y_{n}\right\}_{n \geq 0}$ converges in probability if and only if for every subsequence of joint probabilities laws, $\left\{\mu_{n_{k}, m_{k}}\right\}_{k \geq 0}$, there exists a further subsequence which converges weakly to a probability measure $\mu$ such that

$$
\mu(\{(x, y) \in X \times X: x=y\})=1 .
$$

\section{The Approximation Scheme}

We now implement a Galerkin scheme for (1.1). To this end we introduce the projected operators

$$
B^{n}(U)=P_{n} B(U), \quad F^{n}(U)=P_{n} F(U), \quad \sigma^{n}(U)=P_{n} \sigma(U),
$$

where $U \in V$. We shall also make use of a 'cut-off' function $\theta: \mathbb{R} \rightarrow[0,1]$, which is $C^{\infty}$ and such that:

$$
\theta(x)= \begin{cases}1 & \text { if }|x| \leq \kappa \\ 0 & \text { if }|x| \geq 2 \kappa\end{cases}
$$

Here we choose $\kappa$ to be any positive constant, independent of $n$, such that

$$
\kappa \leq \frac{1}{64 c_{0}}
$$

where $c_{0}$ is the constant appearing in (2.5). The reason for this choice will be made apparent in the proof of Lemma 3.1 (see (3.19), (3.26)).

We now fix a stochastic basis $\mathcal{S}=\left(\Omega, \mathcal{F},\left\{\mathcal{F}_{t}\right\}_{t \geq 0}, \mathbb{P}, W\right)$ and an element $U_{0} \in V$ with law $\mu_{0}$. We find pathwise solutions to the Galerkin systems defined by (3.6) relative to this basis below. Since we allow for an ill-behaved nonlinear term $B$ (that is satisfying $(2.3),(2.4),(2.5))$ we introduce an auxiliary linear system in order to carry uniform estimates on the Galerkin systems. We take $U_{*}^{n}$ to be the unique $\left(H_{n}\right.$ valued) solution of

$$
\frac{d}{d t} U_{*}^{n}+A U_{*}^{n}=0, \quad U_{*}^{n}(0)=P_{n} U_{0}
$$

One may readily verify that, for any $p \geq 2, U_{*}^{n}$ satisfies the estimates

$$
\sup _{t^{\prime} \leq t}\left\|U_{*}^{n}\right\|^{p}+\int_{0}^{T}\left|A U_{*}^{n}\right|^{2}\left\|U_{*}^{n}\right\|^{p-2} d t^{\prime}+\left(\int_{0}^{T}\left|A U_{*}^{n}\right|^{2} d t^{\prime}\right)^{p / 2} \leq c\left\|U_{0}\right\|^{p} .
$$

It is also clear that

$$
\left|U_{*}^{n}\right|_{W^{1,2}(0, T ; H)} \leq c \int_{0}^{T}\left|A U_{*}^{n}\right|^{2} d t^{\prime} \leq c\left\|U_{0}\right\|^{2} .
$$


With these notations in place we define the Galerkin system at order $n$

$$
\begin{gathered}
d U^{n}+\left[A U^{n}+\theta\left(\left\|U^{n}-U_{*}^{n}\right\|\right) B^{n}\left(U^{n}\right)+F^{n}\left(U^{n}\right)\right] d t=\sigma^{n}\left(U^{n}\right) d W, \\
U^{n}(0)=P_{n} U_{0}:=U_{0}^{n} .
\end{gathered}
$$

Here $U^{n}$ is an adapted process in $C\left([0, T] ; H_{n}\right) \cong C\left([0, T], \mathbb{R}^{n}\right)$. The $U_{*}^{n}$ appearing in the cutoff function $\theta$ are solutions of the linear systems (3.3). The significance of this addition will become clear in the proof of Lemma 3.1 below. Note that, due to the preserved cancellation property in the nonlinear portion of the equation, the existence and uniqueness of solutions at each order is standard. See, for example, [16] for further details.

Lemma 3.1. Assume that $F$ and $\sigma$ satifisfy (2.7), (2.10). Fix $U_{0}$, a $V$-valued, $\mathcal{F}_{0}$ measurable random variable and consider the associated sequence of solutions $\left\{U^{n}\right\}_{n \geq 1}$ of the Galerkin system (3.6), (3.3). We suppose that the constant $\kappa$ appearing in the cutoff function $\theta$ satisfies (3.2). Let $p \geq 2$ and suppose that

$$
\mathbb{E}\left\|U_{0}\right\|^{q}<\infty \text { for some } q \geq \max \{2 p, 4\} .
$$

Then there exists a finite number $K>0$ depending only on $p, \mathbb{E}\left\|U_{0}\right\|^{q}$ and the the constants in (2.5), (2.7), (2.10) such that

(i) for every $n \geq 1$,

$$
\mathbb{E}\left(\sup _{t^{\prime} \leq T}\left\|U^{n}\right\|^{p}+\int_{0}^{T}\left|A U^{n}\right|^{2}\left\|U^{n}\right\|^{p-2} d t^{\prime}\right) \leq K,
$$

and also

$$
\mathbb{E}\left(\int_{0}^{T}\left|A U^{n}\right|^{2} d t^{\prime}\right)^{p / 2} \leq K
$$

and finally

$$
\mathbb{E}\left(\left|\int_{0}^{t} \sigma^{n}\left(U^{n}\right) d W\right|_{W^{\alpha, p}([0, T] ; H)}^{p}\right) \leq K
$$

(ii) If under the given assumptions we additionally suppose that $p \geq 4$, then we have, for all $n \geq 1$ :

$$
\mathbb{E}\left(\left|U^{n}(t)-\int_{0}^{t} \sigma^{n}\left(U^{n}\right) d W\right|_{W^{1,2}([0, T] ; H)}^{2}\right) \leq K
$$

Proof. Define $\bar{U}^{n}:=U^{n}-U_{*}^{n}$. We may readily observe that $\bar{U}^{n}$ satisfies

$$
\begin{gathered}
d \bar{U}^{n}+\left[A \bar{U}^{n}+\theta\left(\left\|\bar{U}^{n}\right\|\right) B^{n}\left(\bar{U}^{n}+U_{*}^{n}\right)+F^{n}\left(\bar{U}^{n}+U_{*}^{n}\right)\right] d t=\sigma^{n}\left(\bar{U}^{n}+U_{*}^{n}\right) d W, \\
\bar{U}^{n}(0)=0 .
\end{gathered}
$$

We apply $A^{1 / 2}$ to this system. With the Itō formula we infer, for $p \geq 2$ that,

$$
\begin{aligned}
d\left\|\bar{U}^{n}\right\|^{p} & +p\left|A \bar{U}^{n}\right|^{2}\left\|\bar{U}^{n}\right\|^{p-2} d t \\
= & -p\left\langle F^{n}\left(\bar{U}^{n}+U_{*}^{n}\right), A \bar{U}^{n}\right\rangle\left\|\bar{U}^{n}\right\|^{p-2} d t+\frac{p}{2}\left|\sigma^{n}\left(\bar{U}^{n}+U_{*}^{n}\right)\right|_{L_{2}(\mathfrak{U}, V)}^{2}\left\|\bar{U}^{n}\right\|^{p-2} d t \\
& +\frac{p(p-2)}{2}\left\langle\sigma^{n}\left(\bar{U}^{n}+U_{*}^{n}\right), A \bar{U}^{n}\right\rangle^{2}\left\|\bar{U}^{n}\right\|^{p-4} d t-p \theta\left(\left\|\bar{U}^{n}\right\|\right)\left\langle B^{n}\left(\bar{U}^{n}+U_{*}^{n}\right), A \bar{U}^{n}\right\rangle\left\|\bar{U}^{n}\right\|^{p-2} d t \\
& \quad+p\left\|\bar{U}^{n}\right\|^{p-2}\left\langle\sigma^{n}\left(\bar{U}^{n}+U_{*}^{n}\right), A \bar{U}^{n}\right\rangle d W \\
= & \left(J_{1}^{p}+J_{2}^{p}+J_{3}^{p}+J_{4}^{p}\right) d t+J_{5}^{p} d W .
\end{aligned}
$$


We are able to estimate the first four deterministic terms pointwise in time. Using (2.7) we observe that

$$
\begin{aligned}
\left|J_{1}^{p}\right| & \leq c\left(1+\left\|U_{*}^{n}\right\|+\left\|\bar{U}^{n}\right\|\right)\left|A \bar{U}^{n}\right|\left\|\bar{U}^{n}\right\|^{p-2} \leq \frac{p}{8}\left|A \bar{U}^{n}\right|^{2}\left\|\bar{U}^{n}\right\|^{p-2}+c\left(1+\left\|U_{*}^{n}\right\|+\left\|\bar{U}^{n}\right\|\right)^{2}\left\|\bar{U}^{n}\right\|^{p-2} \\
& \leq \frac{p}{8}\left|A \bar{U}^{n}\right|^{2}\left\|\bar{U}^{n}\right\|^{p-2}+c\left(1+\left\|U_{*}^{n}\right\|\right)^{p}+c\left\|\bar{U}^{n}\right\|^{p} .
\end{aligned}
$$

The terms $J_{2}^{p}$ and $J_{3}^{p}$ are also estimated directly using (2.10)

$$
\left|J_{2}^{p}\right|+\left|J_{3}^{p}\right| \leq c\left(\left(1+\left\|U_{*}^{n}\right\|\right)^{2}+\left\|\bar{U}^{n}\right\|^{2}\right)\left\|\bar{U}^{n}\right\|^{p-2} \leq c\left(1+\left\|U_{*}^{n}\right\|\right)^{p}+c\left\|\bar{U}^{n}\right\|^{p} .
$$

Using the bilinearity of $B$ the term $J_{4}^{p}$ splits according to:

$$
\begin{aligned}
\left|J_{4}^{p}\right| & \leq p \theta\left(\left\|\bar{U}^{n}\right\|\right)\left\|\bar{U}^{n}\right\|^{p-2}\left|A \bar{U}^{n}\right|\left(\left|B\left(U_{*}^{n}\right)\right|+\left|B\left(\bar{U}^{n}, U_{*}^{n}\right)\right|+\left|B\left(U_{*}^{n}, \bar{U}^{n}\right)\right|+\left|B\left(\bar{U}^{n}\right)\right|\right) \\
& :=J_{4,1}^{p}+J_{4,2}^{p}+J_{4,3}^{p}+J_{4,4}^{p} .
\end{aligned}
$$

We estimate each of these terms using (2.5). For $J_{4,1}^{p}$ we have

$$
\begin{aligned}
\left|J_{4,1}^{p}\right| & \leq c_{0} \theta\left(\left\|\bar{U}^{n}\right\|\right)\left\|\bar{U}^{n}\right\|^{p-2}\left|A \bar{U}^{n}\right|\left\|U_{*}^{n}\right\|\left|A U_{*}^{n}\right| \leq \frac{p}{8}\left\|\bar{U}^{n}\right\|^{p-2}\left|A \bar{U}^{n}\right|^{2}+c \theta\left(\left\|\bar{U}^{n}\right\|\right)\left\|\bar{U}^{n}\right\|^{p-2}\left\|U_{*}^{n}\right\|^{2}\left|A U_{*}^{n}\right|^{2} \\
& \leq \frac{p}{8}\left\|\bar{U}^{n}\right\|^{p-2}\left|A \bar{U}^{n}\right|^{2}+c \kappa^{p-2}\left\|U_{*}^{n}\right\|^{2}\left|A U_{*}^{n}\right|^{2} .
\end{aligned}
$$

For the next two terms we estimate

$$
\begin{aligned}
\left|J_{4,2}^{p}\right|+\left|J_{4,3}^{p}\right| & \leq c \theta\left(\left\|\bar{U}^{n}\right\|\right)\left\|\bar{U}^{n}\right\|^{p-2}\left|A \bar{U}^{n}\right|^{3 / 2}\left\|U_{*}^{n}\right\|^{1 / 2}\left|A U_{*}^{n}\right|^{1 / 2}\left\|\bar{U}^{n}\right\|^{1 / 2} \\
& \leq \frac{p}{8}\left\|\bar{U}^{n}\right\|^{p-2}\left|A \bar{U}^{n}\right|^{2}+c \theta\left(\left\|\bar{U}^{n}\right\|\right)\left\|\bar{U}^{n}\right\|^{p}\left\|U_{*}^{n}\right\|^{2}\left|A U_{*}^{n}\right|^{2} \\
& \leq \frac{p}{8}\left\|\bar{U}^{n}\right\|^{p-2}\left|A \bar{U}^{n}\right|^{2}+c \kappa^{p}\left\|U_{*}^{n}\right\|^{2}\left|A U_{*}^{n}\right|^{2} .
\end{aligned}
$$

The last term yields to the bounds

$$
\left|J_{4,4}^{p}\right| \leq c_{0} p \theta\left(\left\|\bar{U}^{n}\right\|\right)\left\|\bar{U}^{n}\right\|^{p-1}\left|A \bar{U}^{n}\right|^{2} \leq 2 \kappa p c_{0}\left\|\bar{U}^{n}\right\|^{p-2}\left|A \bar{U}^{n}\right|^{2} \leq \frac{p}{8}\left\|\bar{U}^{n}\right\|^{p-2}\left|A \bar{U}^{n}\right|^{2} .
$$

Note here that the last inequality follows from the requirement (3.2) imposed on $\kappa$.

Finally we address the stochastic terms. Observe that for any pair of stopping times $0 \leq \tau_{a} \leq \tau_{b} \leq T$, the BDG inequality, (2.12) with $r=1$, implies that

$$
\begin{aligned}
\mathbb{E} \sup _{\tau_{a} \leq t \leq \tau_{b}}\left|\int_{\tau_{a}}^{t} J_{5}^{p} d W\right| & \leq c \mathbb{E}\left(\int_{\tau_{a}}^{\tau_{b}}\left\|\bar{U}^{n}\right\|^{2(p-2)}\left\langle\sigma^{n}\left(\bar{U}^{n}+U_{*}^{n}\right), A \bar{U}^{n}\right\rangle^{2} d s\right)^{1 / 2} \\
& \leq c \mathbb{E}\left(\int_{\tau_{a}}^{\tau_{b}}\left\|\bar{U}^{n}\right\|^{2(p-1)}\left(1+\left\|U_{*}^{n}\right\|+\left\|\bar{U}^{n}\right\|\right)^{2} d s\right)^{1 / 2} \\
& \leq c\left(\mathbb{E} \sup _{\tau_{a} \leq t \leq \tau_{b}}\left\|\bar{U}^{n}\right\|^{p-1}\left(\int_{\tau_{a}}^{\tau_{b}}\left(1+\left\|U_{*}^{n}\right\|+\left\|\bar{U}^{n}\right\|\right)^{2} d s\right)^{1 / 2}\right) \\
& \leq \frac{1}{2} \mathbb{E}\left(\sup _{\tau_{a} \leq t \leq \tau_{b}}\left\|\bar{U}^{n}\right\|^{p}\right)+c \mathbb{E}\left(\int_{\tau_{a}}^{\tau_{b}}\left(1+\left\|U_{*}^{n}\right\|+\left\|\bar{U}^{n}\right\|\right)^{2} d s\right)^{p / 2} \\
& \leq \frac{1}{2} \mathbb{E}\left(\sup _{\tau_{a} \leq t \leq \tau_{b}}\left\|\bar{U}^{n}\right\|^{p}\right)+c \mathbb{E} \int_{\tau_{a}}^{\tau_{b}}\left(\left(1+\left\|U_{*}^{n}\right\|\right)^{p}+\left\|\bar{U}^{n}\right\|^{p}\right) d s .
\end{aligned}
$$

Combining the estimates (3.14), (3.15), (3.16), (3.17), (3.18), (3.19), (3.20) we find, for any $t \in(0, T]$,

$$
\begin{gathered}
\mathbb{E}\left(\sup _{t^{\prime} \in[0, t]}\left\|\bar{U}^{n}\right\|^{p}+\int_{0}^{t}\left|A \bar{U}^{n}\right|^{2}\left\|\bar{U}^{n}\right\|^{p-2} d t^{\prime}\right) \leq c \mathbb{E} \int_{0}^{t}\left(\left\|\bar{U}^{n}\right\|^{p}+\left(1+\left|A U_{*}^{n}\right|^{2}\left\|U_{*}^{n}\right\|^{2}+\left\|U_{*}^{n}\right\|^{p}\right)\right) d t^{\prime} \\
\leq c \int_{0}^{t}\left(\mathbb{E} \sup _{s \in\left[0, t^{\prime}\right]}\left\|\bar{U}^{n}\right\|^{p}+\mathbb{E}\left(1+\left|A U_{*}^{n}\right|^{2}\left\|U_{*}^{n}\right\|^{2}+\left\|U_{*}^{n}\right\|^{p}\right)\right) d t^{\prime} .
\end{gathered}
$$


Applying then the Gronwall inequality yields

$$
\begin{aligned}
\mathbb{E}\left(\sup _{0 \leq t^{\prime} \leq T}\left\|\bar{U}^{n}\right\|^{p}+\int_{0}^{T}\left|A \bar{U}^{n}\right|^{2}\left\|\bar{U}^{n}\right\|^{p-2} d t^{\prime}\right) & \leq c \mathbb{E} \int_{0}^{T}\left(1+\left|A U_{*}^{n}\right|^{2}\left\|U_{*}^{n}\right\|^{2}+\left\|U_{*}^{n}\right\|^{p}\right) d t^{\prime} \\
& \leq c \mathbb{E}\left(1+\left\|U_{0}\right\|\right)^{\max \{p, 4\}} .
\end{aligned}
$$

The second inequality follows from (3.4). We also note that the term involving $\left|A U_{*}^{n}\right|^{2}\left\|U_{*}^{n}\right\|^{2}$ is responsible for the first part of the moment condition (3.7).

In order to complete the proof of (3.8) we observe that

$$
\begin{aligned}
& \mathbb{E}\left(\sup _{0 \leq t^{\prime} \leq T}\left\|U^{n}\right\|^{p}+\int_{0}^{T}\left|A U^{n}\right|^{2}\left\|U^{n}\right\|^{p-2} d t^{\prime}\right) \leq c \mathbb{E}\left(\sup _{0 \leq t^{\prime} \leq T}\left\|U^{n}\right\|^{p}+\left(\int_{0}^{T}\left|A U^{n}\right|^{2} d t^{\prime}\right)^{p / 2}\right) \\
& \leq c \mathbb{E}\left(\sup _{0 \leq t^{\prime} \leq T}\left\|U_{*}^{n}\right\|^{p}+\left(\int_{0}^{T}\left|A U_{*}^{n}\right|^{2} d t^{\prime}\right)^{p / 2}\right)+c \mathbb{E}\left(\sup _{0 \leq t^{\prime} \leq T}\left\|\bar{U}^{n}\right\|^{p}+\left(\int_{0}^{T}\left|A \bar{U}^{n}\right|^{2} d t^{\prime}\right)^{p / 2}\right) .
\end{aligned}
$$

Given the estimates (3.22) for $\bar{U}^{n}$ and (3.4) for $U_{*}^{n}$ it therefore remains to estimate the last term, i.e. to prove the analogue of (3.9) for $\bar{U}$. Returning to (3.13) for the case $p=2$ we must therefore find suitable estimates for the left hand side of the expression

$$
\mathbb{E}\left(\int_{0}^{T}|A \bar{U}|^{2} d t^{\prime}\right)^{p / 2} \leq \mathbb{E}\left(\int_{0}^{T}\left|J_{1}^{2}\right|+\left|J_{2}^{2}\right|+\left|J_{2}^{4}\right| d s+\sup _{t \in[0, T]}\left|\int_{0}^{t} J_{5}^{2} d W\right|\right)^{p / 2} .
$$

Note that, when $p=2,\left|J_{3}^{p}\right|=0$. By treating $\left|J_{1}^{2}\right|,\left|J_{2}^{2}\right|$ in a similar manner to (3.14), (3.15), we infer

$$
\left|J_{1}^{2}\right|+\left|J_{2}^{2}\right| \leq 2^{-(2+2 / p)}\left|A \bar{U}^{n}\right|^{2}+c\left(1+\left\|U_{*}^{n}\right\|^{2}\right)+c\left\|\bar{U}^{n}\right\|^{2} .
$$

For $\left|J_{4}^{2}\right|$ we estimate similarly to (3.16), (3.17), (3.18), (3.19) to deduce

$$
\left|J_{4}^{2}\right| \leq 2^{-(3+2 / p)}\left|A \bar{U}^{n}\right|^{2}+c\left\|U_{*}^{n}\right\|^{2}\left|A U_{*}^{n}\right|^{2}+4 \kappa c_{0}|A \bar{U}|^{2} \leq 2^{-(2+2 / p)}\left|A \bar{U}^{n}\right|^{2}+c\left\|U_{*}^{n}\right\|^{2}\left|A U_{*}^{n}\right|^{2} .
$$

The constant $c_{0}$ after the first inequality is from (2.5). Thus, the assumption (3.2) justifies the second inequality. For the stochastic intergral term in (3.24) we apply the BDG inequality, (2.12), and deduce:

$$
\begin{aligned}
& \mathbb{E} \sup _{t \in[0, T]}\left|\int_{0}^{t} J_{5}^{2} d W\right|^{p / 2} \\
& \leq c \mathbb{E}\left(\int_{0}^{T}\left\langle\sigma^{n}\left(\bar{U}^{n}+U_{*}^{n}\right), A \bar{U}^{n}\right\rangle^{2} d t^{\prime}\right)^{p / 4} \leq c \mathbb{E}\left(\int_{0}^{T}\left(1+\left\|\bar{U}^{n}\right\|^{2}+\left\|U_{*}^{n}\right\|^{2}\right)\left\|\bar{U}^{n}\right\|^{2} d t^{\prime}\right)^{p / 4} \\
& \quad \leq c \mathbb{E}\left(\int_{0}^{T}\left(1+\left\|\bar{U}^{n}\right\|^{4}+\left\|U_{*}^{n}\right\|^{4}\right) d t^{\prime}\right)^{p / 4} \leq c \mathbb{E} \int_{0}^{T}\left(1+\left\|\bar{U}^{n}\right\|^{p}+\left\|U_{*}^{n}\right\|^{p}\right) d t^{\prime} .
\end{aligned}
$$

Applying (3.25), (3.26) and (3.27) to (3.24) we have

$$
\begin{aligned}
& \mathbb{E}\left(\int_{0}^{T}|A \bar{U}|^{2} d t^{\prime}\right)^{p / 2} \\
& \quad \leq \frac{1}{2} \mathbb{E}\left(\int_{0}^{T}|A \bar{U}|^{2} d s\right)^{p / 2}+c \mathbb{E} \int_{0}^{T}\left(1+\left\|U_{*}^{n}\right\|^{p}+\left\|\bar{U}^{n}\right\|^{p}\right) d t^{\prime}+c \mathbb{E}\left(\int_{0}^{T}\left\|U_{*}^{n}\right\|^{2}\left|A U_{*}^{n}\right|^{2} d s\right)^{p / 2} \\
& \quad \leq \frac{1}{2} \mathbb{E}\left(\int_{0}^{T}|A \bar{U}|^{2} d s\right)^{p / 2}+c \mathbb{E} \sup _{t \in[0, T]}\left(1+\left\|U_{*}^{n}\right\|^{p}+\left\|\bar{U}^{n}\right\|^{p}\right)+\mathbb{E}\left\|U_{0}\right\|^{2 p} .
\end{aligned}
$$


Note that the terms involving $\left\|U_{*}^{n}\right\|^{2}\left|A U_{*}^{n}\right|^{2}$ are treated in the final inequality using (3.4) and are responsible for the second part of the moment condition (3.7). Applying (3.28) in turn to (3.23) we finally conclude (3.8). With (3.4), (3.9) also now follows from (3.28)

The bound (3.10) is a direct application of (2.13) with (2.10):

$$
\mathbb{E}\left(\left|\int_{0}^{t} \sigma^{n}\left(U^{n}\right) d W\right|_{W^{\alpha, p}([0, T] ; H)}^{p}\right) \leq c \mathbb{E} \int_{0}^{T}\left|\sigma^{n}\left(U^{n}\right)\right|_{L_{2}(\mathfrak{U}, H)}^{p} d t \leq c \mathbb{E} \int_{0}^{T}\left(1+\left|U^{n}\right|^{p}\right) d t .
$$

We finally establish (3.11). Integrating (3.6) we observe that

$$
U^{n}(t)-\int_{0}^{t} \sigma^{n}\left(U^{n}\right) d W=U_{0}^{n}+\int_{0}^{t}\left[A U^{n}+\theta\left(\left\|U^{n}-U_{*}^{n}\right\|\right) B^{n}\left(U^{n}\right)+F^{n}\left(U^{n}\right)\right] d t .
$$

With, (2.7), (2.5) we infer:

$$
\begin{aligned}
\left|U^{n}(t)-\int_{0}^{t} \sigma^{n}\left(U^{n}\right) d W\right|_{W^{1,2}([0, T] ; H)}^{2} & \leq c\left|U_{0}\right|^{2}+c \int_{0}^{T}\left(\left|A U^{n}\right|^{2}+\left|B^{n}\left(U^{n}\right)\right|^{2}+\left|F^{n}\left(U^{n}\right)\right|^{2}\right) d s \\
& \leq c\left|U_{0}\right|^{2}+c \int_{0}^{T}\left(\left|A U^{n}\right|^{2}+\left|B\left(U^{n}\right)\right|^{2}+\left|F\left(U^{n}\right)\right|^{2}\right) d s \\
& \leq c\left|U_{0}\right|^{2}+c \int_{0}^{T}\left(1+\left\|U^{n}\right\|^{2}\right)\left(1+\left|A U^{n}\right|^{2}\right) d s .
\end{aligned}
$$

Taking expected values in this expression and applying (3.8) (i) for the case $p=4$ gives (3.11). The proof is now complete.

\section{Local Existence of Martingale Solutions}

In this section we establish the existence of a Martingale solution of (1.1). The first step is to make use of the uniform estimates established in Lemma 3.1 we infer the compactness (in certain spaces) of the probability laws associated to the Galerkin approximations. We then change the underlying probabilistic basis in order to find a new sequence of random elements equal in law to the original Galerkin approximations but which converge almost surely. The technical details of the passage to the limit, which is used also below for the case of pathwise solutions, is carried out in Section 7 below.

\subsection{Compactness Arguments}

For a given initial distribution $\mu_{0}$ on $V$ we fix a stochastic basis $\mathcal{S}=\left(\Omega, \mathcal{F},\left\{\mathcal{F}_{t}\right\}_{t \geq 0}, \mathbb{P}, W\right)$ upon which is defined an $\mathcal{F}_{0}$ measurable random element $U_{0}$ with distribution $\mu_{0}$. Consider the sequence of Galerkin approximations $\left\{U^{n}\right\}$ solving (3.6) relative to this basis and initial condition. We consider the phase space:

$$
\mathcal{X}_{U}=\left(L^{2}(0, T ; V) \cap C\left([0, T], V^{\prime}\right), \quad \mathcal{X}_{W}=C\left([0, T], \mathfrak{U}_{0}\right), \quad \mathcal{X}=\mathcal{X}_{U} \times \mathcal{X}_{W}\right.
$$

We may think of the first component, $\mathcal{X}_{U}$, of this phase space as the set where the solution $U^{n}$ lives and the second component, $\mathcal{X}_{W}$, as being the set on which the driving Brownian motions are defined. We consider the probability measures

$$
\mu_{U}^{n}(\cdot)=\mathbb{P}\left(U^{n} \in \cdot\right) \in \operatorname{Pr}\left(L^{2}([0, T] ; V) \cap C\left([0, T], V^{\prime}\right)\right)
$$

and

$$
\mu_{W}(\cdot)=\mu_{W}^{n}(\cdot)=\mathbb{P}(W \in \cdot) \in \operatorname{Pr}\left(C\left([0, T], \mathfrak{U}_{0}\right)\right)
$$

This defines a sequence of probability measures $\mu^{n}=\mu_{U}^{n} \times \mu_{W}^{n}$ on the phase space $\mathcal{X}$. By making appropiate use of Lemma 3.1 we will now show that this sequence is tight. More precisely: 
Lemma 4.1. Suppose that $\mu_{0}$ satisfies (2.16) with $q \geq 8$. Consider the measures $\mu^{n}$ on $\mathcal{X}$ defined according to (4.2), (4.3). Then the sequence $\left\{\mu^{n}\right\}_{n \geq 1}$ is tight and therefore weakly compact over the phase space $\mathcal{X}$.

Proof. By applying Lemma 2.2, (i) with $X_{-1}=H, X_{0}=V, X_{1}=D(A), p=2$ and $\alpha=1 / 4$ we deduce that

$$
L^{2}([0, T] ; D(A)) \cap W^{1 / 4,2}([0, T] ; H) \subset \subset L^{2}([0, T] ; V) .
$$

For $R>0$ we define the set

$$
B_{R}^{1}=\left\{U \in L^{2}([0, T] ; D(A)) \cap W^{1 / 4,2}([0, T] ; H):|U|_{L^{2}([0, T] ; D(A))}^{2}+|U|_{W^{1 / 4,2}([0, T] ; H)}^{2} \leq R^{2}\right\}
$$

which is thus compact in $L^{2}([0, T], V)$. Due to the Chebyshev inequality and the uniform estimates (3.8), (3.11), (3.10) in the case $p=2$, we estimate,

$$
\begin{aligned}
\mu_{U}^{n}\left(\left(B_{R}^{1}\right)^{C}\right) & =\mathbb{P}\left(\left|U^{n}\right|_{L^{2}([0, T] ; D(A))}^{2}+\left|U^{n}\right|_{W^{1 / 4,2}([0, T] ; H)}^{2} \geq R^{2}\right) \\
& \leq \mathbb{P}\left(\left|U^{n}\right|_{L^{2}([0, T] ; D(A))}^{2} \geq R^{2} / 2\right)+\mathbb{P}\left(\left|U^{n}\right|_{W^{1 / 4,2}([0, T] ; H)}^{2} \geq R^{2} / 2\right) \\
& \leq \frac{2}{R^{2}} \mathbb{E}\left(\int_{0}^{T}\left|A U^{n}\right|^{2} d t^{\prime}+\left|U^{n}\right|_{W^{\frac{1}{4}, 2}([0, T] ; H)}^{2}\right) \leq \frac{c}{R^{2}},
\end{aligned}
$$

where the numerical constant $c$ is independent of $n$.

Choose $\alpha \in(1 / q, 1 / 2)$ so that $\alpha q>1$. By Lemma 2.2, (ii) with $Y_{0}=V^{\prime}=D\left(A^{-1 / 2}\right)$ and $Y=H$ we infer the compact embeddings

$$
W^{1,2}([0, T] ; H) \subset \subset C\left([0, T], V^{\prime}\right), \quad W^{\alpha, q}([0, T] ; H) \subset \subset C\left([0, T], V^{\prime}\right) .
$$

For $R>0$, we take $B_{R}^{2,1}$ and $B_{R}^{2,2}$ to be the balls of radius $R$ in $W^{1,2}([0, T], H)$ and $W^{\alpha, q}([0, T], H)$ respectively. It follows that for $R>0, B_{R}^{2}:=B_{R}^{2,1}+B_{R}^{2,2}$ is compact in $C\left([0, T], V^{\prime}\right)$. Since indeed,

$$
\left\{U^{n} \in B_{R}^{2}\right\} \supset\left\{U^{n}(t)-\int_{0}^{t} \sigma^{n}\left(U^{n}\right) d W \in B_{R}^{2,1}\right\} \cap\left\{\int_{0}^{t} \sigma^{n}\left(U^{n}\right) d W \in B_{R}^{2,2}\right\},
$$

we may apply Chebyshev's inequality and then the uniform estimates (3.11) (3.10) to infer

$$
\begin{aligned}
\mu_{U}^{n}\left(\left(B_{R}^{2}\right)^{C}\right) \leq & \mathbb{P}\left(\left|U^{n}(t)-\int_{0}^{t} \sigma^{n}\left(U^{n}\right) d W\right|_{W^{1,2}([0, T] ; H)}^{2} \geq R^{2}\right) \\
& +\mathbb{P}\left(\left|\int_{0}^{t} \sigma^{n}\left(U^{n}\right) d W\right|_{W^{\alpha, q}([0, T] ; H)}^{q} \geq R^{q}\right) \\
\leq & \frac{c}{R^{2}} .
\end{aligned}
$$

As above the $c$ is independent of $n$.

It is not hard to see ${ }^{2}$ that $B_{R}^{1} \cap B_{R}^{2}$ is compact in $L^{2}(0, T ; V) \cap C\left([0, T], V^{\prime}\right)$ for every $R>0$. As a consequence of (4.4) and (4.5) we have

$$
\mu_{U}^{n}\left(\left(B_{R}^{1} \cap B_{R}^{2}\right)^{C}\right) \leq \mu_{U}^{n}\left(\left(B_{R}^{1}\right)^{C}\right)+\mu_{U}^{n}\left(\left(B_{R}^{2}\right)^{C}\right) \leq \frac{c}{R^{2}}
$$

We therefore take $A_{\epsilon}:=B_{\sqrt{2 c / \epsilon}}^{1} \cap B_{\sqrt{2 c / \epsilon}}^{2}$, with $c$ the constant which appears on the left hand side immediately above. With this definition we infer that for $\epsilon>0$,

$$
\mu_{U}^{n}\left(A_{\epsilon}\right) \geq 1-\frac{\epsilon}{2}
$$

\footnotetext{
${ }^{2}$ One need only verify that if $\left\{U^{n}\right\}_{n \geq 0} \subset L^{2}(0, T ; V) \cap C\left([0, T], V^{\prime}\right)$ and if $U^{n} \rightarrow U$ in $L^{2}(0, T ; V), U^{n} \rightarrow \tilde{U}$ in $C\left([0, T], V^{\prime}\right)$ that $U=\tilde{U}$
} 
over all $n$.

We next turn to the sequence $\left\{\mu_{W}^{n}\right\}_{n \geq 0}$. This sequence is constantly equal to one element and is thus weakly compact. Hence, as a consequence of Proposition 2.1, (i) $\left\{\mu_{W}^{n}\right\}_{n \geq 0}$ must be tight. We therefore infer the existence of collection of compact sets $\tilde{A}_{\epsilon} \subset C\left([0, T], \mathfrak{U}_{0}\right)$ so that

$$
\mu_{W}^{n}\left(\tilde{A}_{\epsilon}\right) \geq 1-\frac{\epsilon}{2}
$$

for all $n$.

We now have everything in hand to conclude the tightness and therefore the weak compactness of $\left\{\mu^{n}\right\}_{n \geq 0}$. For $\epsilon>0$ we define $\mathcal{K}_{\epsilon}:=A_{\epsilon} \times \tilde{A}_{\epsilon}$ which are compact in $\mathcal{X}$. By (4.6) and (4.7) we infer that, for any $\epsilon>0$ and every $n$,

$$
\mu^{n}\left(\mathcal{K}_{\epsilon}\right) \geq 1-\epsilon
$$

and thus that $\left\{\mu^{n}\right\}_{n \geq 0}$ is tight in $\mathcal{X}$. Prohorov's theorem, given herein as Proposition 2.1 therefore implies that $\mu^{n}$ is weakly compact. The proof is therefore complete.

\subsubsection{Strong Convergence on the Skorohod Space}

Given $\mu_{0}$ (satisfying (2.16) with $q \geq 8$ ) we have shown that the sequence of measures $\left\{\mu^{n}\right\}_{n \geq 1}$ associated to the Galerkin sequence $\left(U^{n}, W\right)$ are weakly compact on $\mathcal{X}$. Passing to a weakly convergent subsequence $\mu^{n_{k}}$ we now apply the Skorohod embedding theorem, Proposition 2.1, to infer the following Proposition.

Proposition 4.1. Suppose that $\mu_{0}$ is a probability measure on $V$ satisfying (2.16) with $p>4$. Then there exists a probability space $(\tilde{\Omega}, \tilde{\mathcal{F}}, \tilde{\mathbb{P}})$ and a subsequence $n_{k}$ and a sequence of $\mathcal{X}$ valued random variables $\left(\tilde{U}^{n_{k}}, \tilde{W}^{n_{k}}\right)$ such that

(i) $\left(\tilde{U}^{n_{k}}, \tilde{W}^{n_{k}}\right)$ converges almost surely, in the topology of $\mathcal{X}$, to an element $(\tilde{U}, \tilde{W})$.

(ii) $\tilde{W}^{n_{k}}$ is a cylindrical Wiener process, relative to the filtration $\mathcal{F}_{t}^{m_{k}}$, given by the completion of $\sigma\left(\tilde{W}^{m_{k}}(s)\right.$, $\left.\tilde{U}^{m_{k}}(s) ; s \leq t\right)$.

(iii) Each pair $\left(\tilde{U}^{n_{k}}, \tilde{W}^{n_{k}}\right)$ satisfies

$$
\begin{gathered}
d \tilde{U}^{n_{k}}+\left[A \tilde{U}^{n_{k}}+\theta\left(\left\|\tilde{U}^{n_{k}}-\tilde{U}_{*}^{n_{k}}\right\|\right) B^{n_{k}}\left(\tilde{U}^{n_{k}}\right)+F^{n_{k}}\left(\tilde{U}^{n_{k}}\right)\right] d t=\sigma^{n_{k}}\left(\tilde{U}^{n_{k}}\right) d \tilde{W}^{n_{k}}, \\
\tilde{U}^{n_{k}}(0)=P_{n_{k}} \tilde{U}(0)^{n_{k}}:=\tilde{U}_{0}^{n_{k}},
\end{gathered}
$$

where we define $\tilde{U}_{*}^{n_{k}}$ by:

$$
\frac{d}{d t} \tilde{U}_{*}^{n_{k}}+A \tilde{U}_{*}^{n_{k}}=0 \quad \tilde{U}_{*}^{n_{k}}(0)=\tilde{U}_{0}^{n_{k}} .
$$

With this proposition established the existence of a local Martingale solution follows once we have shown that $(\tilde{U}, \tilde{W})$ and an appropriately defined stopping time $\tau$ (see (7.5)) satisfy (1.1). This passage to the limit argument, which is technical and delicate, is carried out in Section 7 below.

Remark 4.1. While Proposition 4.1, (i) follows directly from Proposition 2.1, (ii) further steps are required to establish (ii), (iii). These technical points may be demonstrated in a similar manner to previous works. See [1].

\section{Local Pathwise Solutions}

We turn now to study Pathwise solutions of (1.1). Here the key step is to apply Proposition 2.2 in order to show that $\left(U^{n}, W\right)$ converges almost surely in $L^{2}([0, T] ; V) \cap C\left([0, T], V^{\prime}\right)$ relative to the initial stochastic basis. The diagonal condition, (2.26) translates to a question of pathwise uniqueness which we address first. 


\subsection{Local Pathwise Uniqueness}

The following proposition establishes the uniqueness, pathwise, for any pair of solutions of the modified system (2.22). Such solutions appear in an intermediate step in the compactness arguments in Section 5.2 below.

Proposition 5.1. Suppose that $\left(\mathcal{S}, U^{(1)}\right)$ and $\left(\mathcal{S}, U^{(2)}\right)$ are two global Martingale solutions of $(2.22)$ relative to the same stochastic basis $\mathcal{S}:=\left(\Omega, \mathcal{F},\left\{\mathcal{F}_{t}\right\}_{t \geq 0}, \mathbb{P}, W\right)$. Assume that, in addition to the conditions imposed in Definition 2.1, $F$ and $\sigma$ satisfy the Lipschitz conditions (2.8) and (2.11). Define

$$
\Omega_{0}=\left\{U^{(1)}(0)=U^{(2)}(0)\right\} .
$$

Then $U^{(1)}$ and $U^{(2)}$ are indistinguishable on $\Omega_{0}$ in the sense that

$$
\mathbb{P}\left(\mathbb{1}_{\Omega_{0}}\left(U^{1}(t)-U^{2}(t)=0 ; \forall t \geq 0\right)=1 .\right.
$$

Remark 5.1. We note that, with trivial modifications to the proof that follows, one may establish that Pathwise solutions of (1.1) are unique in the sense of Definition 2.2, (ii).

Proof of Proposition 5.1. Define $R=U^{(1)}-U^{(2)}$ and let $\bar{R}=\mathbb{1}_{\Omega_{0}} R$. Note that, by definition, $\bar{R} \in$ $C([0, \infty) ; V) \cap L_{\text {loc }}^{2}([0, \infty) ; D(A))$, a.s. Due to the bilinear term $B$, when we attempt to estimate $\bar{R}$, stray terms arise that involve only $U^{(1)}$ or $U^{(2)}$. See $(5.8),(5.9)$ below. To remedy this situation we define the stopping times

$$
\tau^{(n)}:=\inf _{t \geq 0}\left\{\int_{0}^{t}\left\|U^{(1)}\right\|^{2}\left|A U^{(1)}\right|^{2}+\left\|U^{(2)}\right\|^{2}\left|A U^{(2)}\right|^{2} d s \geq n\right\}
$$

Clearly this is an increasing sequence. Futhermore, since $U^{(1)}, U^{(2)}$ are global solutions, we may infer that $\lim _{n \rightarrow \infty} \tau^{(n)}=\infty$ from (2.18). Hence, the desired result will follow if we show that for any $n, T>0$,

$$
\mathbb{E}\left(\sup _{\left[0, \tau^{(n)} \wedge T\right]}\|\bar{R}\|^{2}\right)=0
$$
$R$

Subtracting the equations (c.f. $(2.22))$ for $U^{(2)}$ from that for $U^{(1)}$ we arrive at the following equation for

$$
\begin{aligned}
d R+(A R & +\theta\left(\left\|U^{(1)}-U_{*}^{(1)}\right\|\right) B\left(U^{(1)}\right)-\theta\left(\left\|U^{(2)}-U_{*}^{(2)}\right\|\right) B\left(U^{(2)}\right) \\
& \left.+F\left(U^{(1)}\right)-F\left(U^{(2)}\right)\right) d t=\left(\sigma\left(U^{(1)}\right)-\sigma\left(U^{(2)}\right)\right) d W \\
R(0) & =U^{(1)}(0)-U^{(2)}(0)
\end{aligned}
$$

Itō's lemma yields the following evolution equation for $\|R\|^{2}$ :

$$
\begin{aligned}
d\|R\|^{2}+2|A R|^{2}= & 2\left\langle\theta\left(\left\|U^{(2)}-U_{*}^{(2)}\right\|\right) B\left(U^{(2)}\right)-\theta\left(\left\|U^{(1)}-U_{*}^{(1)}\right\|\right) B\left(U^{(1)}\right), A R\right\rangle \\
& +2\left\langle F\left(U^{(2)}\right)-F\left(U^{(1)}\right), A R\right\rangle d t+\left\|\sigma\left(U^{(1)}\right)-\sigma\left(U^{(2)}\right)\right\|_{L_{2}(\mathfrak{U}, V)}^{2} d t \\
& +2\left\langle\sigma\left(U^{(1)}\right)-\sigma\left(U^{(2)}\right), A R\right\rangle d W .
\end{aligned}
$$

Fix $n$ and stopping times $\tau_{a}, \tau_{b}$, such that $0 \leq \tau_{a} \leq \tau_{b} \leq \tau^{(n)}$. Integrating in time and taking supremums, 
multiplying by $\mathbb{1}_{\Omega_{0}}$ and finally taking an expected value we arrive at the expression

$$
\begin{aligned}
& \mathbb{E}\left(\sup _{t \in\left[\tau_{a}, \tau_{b}\right]}\|\bar{R}\|^{2}+\int_{\tau_{a}}^{\tau_{b}}|A \bar{R}|^{2} d s\right) \\
& \leq \mathbb{E}\left\|\bar{R}\left(\tau_{a}\right)\right\|^{2}+2 \mathbb{E} \int_{\tau_{a}}^{\tau_{b}}\left|\left\langle\left(\theta\left(\left\|U^{(1)}-U_{*}^{(1)}\right\|\right)-\theta\left(\left\|U^{(2)}-U_{*}^{(2)}\right\|\right)\right) B\left(U^{(1)}\right), A \bar{R}\right\rangle\right| d t \\
& \quad+2 \mathbb{E} \int_{\tau_{a}}^{\tau_{b}}\left|\left\langle B\left(U^{(1)}\right)-B\left(U^{(2)}\right), A \bar{R}\right\rangle\right| d t+2 \mathbb{E} \int_{\tau_{a}}^{\tau_{b}}\left|\left\langle F\left(U^{(1)}\right)-F\left(U^{(2)}\right), A \bar{R}\right\rangle\right| d t \\
& \quad+2 \mathbb{E} \sup _{t \in\left[\tau_{a}, \tau_{b}\right]}\left|\int_{\tau_{a}}^{t}\left\langle\sigma\left(U^{(1)}\right)-\sigma\left(U^{(2)}\right), A \bar{R}\right\rangle d W\right|+\mathbb{E} \int_{\tau_{a}}^{\tau_{b}} \mathbb{1}_{\Omega_{0}}\left\|\sigma\left(U^{(1)}\right)-\sigma\left(U^{(2)}\right)\right\|_{L_{2}(\mathfrak{U}, V)}^{2} d s \\
& :=\mathbb{E}\left\|\bar{R}\left(\tau_{a}\right)\right\|^{2}+J_{1}+J_{2}+J_{3}+J_{4}+J_{5} .
\end{aligned}
$$

For $J_{1}$, we use that $\theta$ is Lipschitz. See (3.1). Applying (2.5), we have

$$
\begin{aligned}
J_{1} & \leq c \mathbb{E} \int_{\tau_{a}}^{\tau_{b}}\left\|\left(U^{(1)}-U^{(2)}\right)-\left(U_{*}^{(1)}-U_{*}^{(2)}\right)\right\|\left|\left\langle B\left(U^{(1)}\right), A \bar{R}\right\rangle\right| d t \leq c \mathbb{E} \int_{\tau_{a}}^{\tau_{b}}\|\bar{R}\|\left\|U^{(1)}\right\|\left|A U^{(1)} \| A \bar{R}\right| d t \\
& \leq \frac{1}{4} \mathbb{E} \int_{\tau_{a}}^{\tau_{b}}|A \bar{R}|^{2} d s+c \mathbb{E} \int_{\tau_{a}}^{\tau_{b}}\left\|U^{(1)}\right\|^{2}\left|A U^{(1)}\right|^{2}\|\bar{R}\|^{2} d s .
\end{aligned}
$$

Note that since both $U_{*}^{(1)}, U_{*}^{(2)}$ satisfy the linear equation (2.23) it is clear that, for every $t \geq 0 \mathbb{1}_{\Omega_{0}}\left(U_{*}^{(1)}(t)-\right.$ $\left.U_{*}^{(2)}(t)\right)=0$ almost surely. For $J_{2}$ the bilinearity of $B$ and (2.5) imply:

$$
\begin{aligned}
J_{2}= & 2 \mathbb{E} \int_{\tau_{a}}^{\tau_{b}}\left|\left\langle B\left(U^{(1)}-U^{(2)}, U^{(1)}\right)+B\left(U^{(2)}, U^{(1)}-U^{(2)}\right), A \bar{R}\right\rangle\right| d t \\
& \leq 2 \mathbb{E} \int_{\tau_{a}}^{\tau_{b}}\left|\left\langle B\left(\bar{R}, U^{(1)}\right)+B\left(U^{(2)}, \bar{R}\right), A \bar{R}\right\rangle\right| d t \\
& \leq c \mathbb{E} \int_{\tau_{a}}^{\tau_{b}}\left(\left\|U^{(1)}\right\|^{1 / 2}\left|A U^{(1)}\right|^{1 / 2}+\left\|U^{(2)}\right\|^{1 / 2}\left|A U^{(2)}\right|^{1 / 2}\right)\|\bar{R}\|^{1 / 2}|A \bar{R}|^{3 / 2} d s \\
& \leq \frac{1}{4} \mathbb{E} \int_{\tau_{a}}^{\tau_{b}}|A \bar{R}|^{2} d s+c \mathbb{E} \int_{\tau_{a}}^{\tau_{b}}\left(\left\|U^{(1)}\right\|^{2}\left|A U^{(1)}\right|^{2}+\left\|U^{(2)}\right\|^{2}\left|A U^{(2)}\right|^{2}\right)\|\bar{R}\|^{2} d s .
\end{aligned}
$$

The terms $J_{3}$ and $J_{5}$ are estimated directly making use of (2.8) to infer

$$
\begin{aligned}
J_{3} & \leq c \mathbb{E} \int_{\tau_{a}}^{\tau_{b}} \mathbb{1}_{\Omega_{0}}\left|F\left(U^{(1)}\right)-F\left(U^{(2)}\right) \| A \bar{R}\right| d s \\
& \leq c \mathbb{E} \int_{\tau_{a}}^{\tau_{b}}\|\bar{R}\||A \bar{R}| d s \leq \frac{1}{4} \mathbb{E} \int_{\tau_{a}}^{\tau_{b}}|A \bar{R}|^{2} d s+c \mathbb{E} \int_{\tau_{a}}^{\tau_{b}}\|\bar{R}\|^{2} d s,
\end{aligned}
$$

and making use of (2.11) to deduce

$$
J_{5} \leq c \mathbb{E} \int_{\tau_{a}}^{\tau_{b}}\|\bar{R}\|^{2} d s
$$

Finally, $J_{4}$ is addressed using (2.12), with $r=1$ and then (2.11)

$$
\begin{aligned}
J_{4} & \leq c \mathbb{E}\left(\int_{\tau_{a}}^{\tau_{b}} \mathbb{1}_{\Omega_{0}}\left\langle\sigma\left(U^{(1)}\right)-\sigma\left(U^{(2)}\right), A \bar{R}\right\rangle^{2} d s\right)^{1 / 2} \\
& \leq c \mathbb{E}\left(\int_{\tau_{a}}^{\tau_{b}} \mathbb{1}_{\Omega_{0}}\left\|\sigma\left(U^{(1)}\right)-\sigma\left(U^{(2)}\right)\right\|_{L_{2}(\mathfrak{U}, V)}^{2}\|\bar{R}\|^{2} d s\right)^{1 / 2} \\
& \leq c \mathbb{E}\left(\int_{\tau_{a}}^{\tau_{b}}\|\bar{R}\|^{4}\right)^{1 / 2} \leq \frac{1}{2} \mathbb{E} \sup _{t \in\left[\tau_{a}, \tau_{b}\right]}\|\bar{R}\|^{2}+c \mathbb{E} \int_{\tau_{a}}^{\tau_{b}}\|\bar{R}\|^{2} d s .
\end{aligned}
$$


Applying the estimates in (5.8), (5.9), (5.10), (5.11), (5.12) to (5.7) we infer

$$
\begin{aligned}
\mathbb{E}\left(\sup _{t \in\left[\tau_{a}, \tau_{b}\right]}\|\bar{R}\|^{2}+\int_{\tau_{a}}^{\tau_{b}}|A \bar{R}|^{2} d s\right) \\
\quad \leq c \mathbb{E}\left\|\bar{R}\left(\tau_{a}\right)\right\|^{2}+c \mathbb{E} \int_{\tau_{a}}^{\tau_{b}}\left(\left\|U^{(1)}\right\|^{2}\left|A U^{(1)}\right|^{2}+\left\|U^{(2)}\right\|^{2}\left|A U^{(2)}\right|^{2}+1\right)\|\bar{R}\|^{2} d s .
\end{aligned}
$$

With this estimate we may finally apply the stochastic Gronwall lemma, as in [23] to conclude (5.4). The proof is complete.

\subsection{Compactness Revisited}

We return to the sequence $\left\{U^{n}\right\}$ of Galerkin solutions of (3.6) defined relative to the given stochastic basis $\mathcal{S}$. We assume throughout this section that $\mathbb{E}\left\|U_{0}\right\|^{q}<\infty$ for some $q \geq 8$. Once we have established the existence of local pathwise solutions for all initial data in this class the general case, (2.17) may be established via a localization argument. See e.g. [23].

In pursuit of Proposition 2.2 we consider the collection of joint distributions $\mu_{U}^{n, m}$ given by $\left(U^{n}, U^{m}\right)$. For this purpose we define the extended phase space (cf. (4.1))

$$
\begin{array}{r}
\mathcal{X}^{J}=\mathcal{X}_{U} \times \mathcal{X}_{U} \times \mathcal{X}_{W}, \quad \mathcal{X}_{U}^{J}:=\mathcal{X}_{U} \times \mathcal{X}_{U}, \\
\mathcal{X}_{U}=L^{2}(0, T ; V) \cap C\left([0, T], V^{\prime}\right), \quad \mathcal{X}_{W}=C\left([0, T], \mathfrak{U}_{0}\right) .
\end{array}
$$

As above in (4.2), (4.3) we let $\mu_{U}^{n}(E)=\mathbb{P}\left(U^{n} \in E\right)$ for $E \in \mathcal{X}_{U}$ and $\mu_{W}(E)=\mathbb{P}(W \in E)$ for $E \in \mathcal{X}_{W}$. Take

$$
\mu_{U}^{n, m}=\mu_{U}^{n} \times \mu_{U}^{m}, \quad \nu^{n, m}=\mu_{U}^{n} \times \mu_{U}^{m} \times \mu_{W} .
$$

Similarly to Lemma 4.1 we prove:

Lemma 5.1. Suppose $\mathbb{E}\left\|U_{0}\right\|^{q}<\infty$ for some $q \geq 8$. The collection $\left\{\nu^{n, m}\right\}$ (and hence any subsequence $\left\{\nu^{n_{k}, m_{k}}\right\}$ ) is tight (and hence compact) on $\mathcal{X}^{J}$.

Proof. The proof is nearly identical to Lemma 4.1. We determine the sets $B_{R}^{1}, B_{R}^{2}$ exactly as previously. With trivial modifications (see (4.6) and remarks immediately above) we can therefore choose $A_{\epsilon}, \tilde{A}_{\epsilon}$ compact in $\mathcal{X}_{U}$ and $\mathcal{X}_{W}$ respectively so that $\mu_{U}^{n}\left(A_{\epsilon}\right) \geq 1-\frac{\epsilon}{4}$, and $\mu_{W}^{n}\left(\tilde{A}_{\epsilon}\right) \geq 1-\frac{\epsilon}{2}$, for every $n$. Taking $\mathcal{K}_{\epsilon}:=A_{\epsilon} \times A_{\epsilon} \times \tilde{A}_{\epsilon}$, which is compact in $\mathcal{X}^{J}$ we see that $\nu^{n, m}\left(\mathcal{K}_{\epsilon}\right) \geq\left(1-\frac{\epsilon}{4}\right)^{2}\left(1-\frac{\epsilon}{2}\right) \geq 1-\epsilon$, which holds for every $0<\epsilon<1$ over all $m, n$. The proof is complete.

Suppose now that $\left\{\nu_{U}^{n_{k}, m_{k}}\right\}_{k \geq 0}$ is any subsequence. By Lemma 5.1, $\left\{\nu_{U}^{n_{k}, m_{k}}\right\}_{k \geq 0}$ is tight and hence by Proposition 2.1 (i), we may choose as subsequence $k^{\prime}$ so that $\nu^{n_{k}^{\prime}, m_{k}^{\prime}}$ converges weakly to an element $\nu^{\prime}$. By applying Proposition 2.1, (ii) we next infer the existence of a probability space $(\tilde{\Omega}, \tilde{\mathcal{F}}, \tilde{\mathbb{P}})$ upon which are defined a sequence of random elements $\left(\tilde{U}^{n_{k}^{\prime}}, \tilde{\tilde{U}}^{m_{k}^{\prime}}, \tilde{W}^{k^{\prime}}\right)$ converging almost surely in $\mathcal{X}^{J}$ to an element $(\tilde{U}, \tilde{\tilde{U}}, \tilde{W})$ in such a way that $\tilde{\mathbb{P}}\left(\left(\tilde{U}^{n_{k}^{\prime}}, \tilde{\tilde{U}} m_{k}^{\prime}, \tilde{W}^{k^{\prime}}\right) \in \cdot\right)=\nu^{n_{k}^{\prime}, m_{k}^{\prime}}(\cdot)$ and $\tilde{\mathbb{P}}((\tilde{U}, \tilde{\tilde{U}}, \tilde{W}) \in \cdot)=\nu^{\prime}(\cdot)$. Let $\tilde{Z}_{k^{\prime}}=\left(\tilde{U}^{n_{k}^{\prime}}, \tilde{W}^{k^{\prime}}\right), \tilde{\tilde{Z}}_{k^{\prime}}=\left(\tilde{\tilde{U}}^{n_{k}^{\prime}}, \tilde{W}^{k^{\prime}}\right), \tilde{Z}=(\tilde{U}, \tilde{W})$ and $\tilde{Z}=(\tilde{\tilde{U}}, \tilde{W})$. Note that in particular $\mu_{U}^{n_{k}, m_{k}}$ converges weakly to the measure $\mu_{U}$ defined by

$$
\mu_{U}(\cdot):=\tilde{\mathbb{P}}((\tilde{U}, \tilde{\tilde{U}}) \in \cdot)
$$

Exactly as for Proposition 4.1, we may establish the conditions for Proposition 7.1 below for both $\tilde{Z}_{k^{\prime}}$, $\tilde{Z}$ and $\tilde{\tilde{Z}}_{k^{\prime}}, \tilde{\tilde{Z}}$. As such we infer that both $\tilde{U}$ and $\tilde{\tilde{U}}$ are global Martingale solutions of $(2.22)$ over the same stochastic basis $\tilde{S}=\left(\Omega, \mathcal{F},\left\{\mathcal{F}_{t}\right\}_{t>0}, \mathbb{P}, \tilde{W}\right)$. Since it may be readily shown from the above convergences that $\tilde{U}(0)=\tilde{\tilde{U}}(0)$ a.s. we infer from Proposition 5.1 that $\tilde{U}=\tilde{\tilde{U}}$ in $\mathcal{X}_{U}$ a.s. In other words

$$
\mu\left(\left\{(x, y) \in \mathcal{X}_{U}^{J} \times \mathcal{X}_{U}^{J}: x=y\right\}\right)=\tilde{\mathbb{P}}\left(\tilde{U}=\tilde{\tilde{U}} \text { in } \mathcal{X}_{U}\right)=1 .
$$


With this conclusion, Proposition 2.2, now implies that the original sequence $U^{n}$ defined on the initial probability space $(\Omega, \mathcal{F}, \mathbb{P})$ converges to an element $U$, in the topology of $\mathcal{X}_{U}$. By a final application of Proposition $7.1^{3}$ below we may infer that $U$ is a global pathwise solution of (2.22). Hence, taking $\tau$ as in (7.5) below we conclude that $(U, \tau)$ is a local pathwise solution of (1.1).

The passage from a local to a maximal pathwise solution in the sense of Definition 2.2, (iii), may now be carried out as in [26]. See also [29]. This completes the proof of Theorem 2.1.

\section{Example: The Primitive Equations of the Ocean}

As discussed in the introduction the primary motivation in the development of the abstract theory was to be able to treat the existence of local, pathwise solutions of the stochastic primitive equations of the ocean considered in the $\beta$-plane approximation. We now recall this system of equations and show how Theorem 2.1 applies to these equations.

The stochastic primitive equations of the ocean in their $\beta$-plane approximation take the form

$$
\begin{gathered}
\partial_{t} \mathbf{v}+(\mathbf{v} \cdot \nabla) \mathbf{v}+w \partial_{z} \mathbf{v}+\frac{1}{\rho_{0}} \nabla p+f \mathbf{k} \times \mathbf{v}-\mu_{\mathbf{v}} \Delta \mathbf{v}-\nu_{\mathbf{v}} \partial_{z z} \mathbf{v}=F_{\mathbf{v}}+\sigma_{\mathbf{v}}(\mathbf{v}, T, S) \dot{W}_{1}, \\
\partial_{z} p=-\rho g, \\
\nabla \cdot \mathbf{v}+\partial_{z} w=0, \\
\partial_{t} T+(\mathbf{v} \cdot \nabla) T+w \partial_{z} T-\mu_{T} \Delta T-\nu_{T} \partial_{z z} T=F_{T}+\sigma_{T}(\mathbf{v}, T, S) \dot{W}_{2}, \\
\partial_{t} S+(\mathbf{v} \cdot \nabla) S+w \partial_{z} S-\mu_{S} \Delta S-\nu_{S} \partial_{z z} S=F_{S}+\sigma_{S}(\mathbf{v}, T, S) \dot{W}_{3}, \\
\rho=\rho_{0}\left(1-\beta_{T}\left(T-T_{r}\right)+\beta_{S}\left(S-S_{r}\right)\right) .
\end{gathered}
$$

Here, $\mathbf{v}, T, S, p, \rho$ represent the horizontal velocity,temperature, salt concentration, pressure and density of the fluid under consideration; $\mu_{\mathbf{v}}, \nu_{\mathbf{v}}, \mu_{T}, \nu_{T}, \mu_{S}, \nu_{S}$ are (possibly anisotropic) coefficients of the eddy viscosity and of the heat and salt diffusivity respectively; $f$ is the Coriolis parameter appearing in the antisymmetric term in (6.1) and accounts for the earth's rotation in the momentum budget. The stochastic terms are driven by white noise processes $\dot{W}_{j}$ and are understood in the Itō sense. The equations as given above model oceanic flows, however equations of a quite similar structure may be given that describe the atmosphere and the coupled oceanic atmospheric system, or the same equations on the sphere.

Of course (6.1) is supplemented with appropriate boundary conditions which, among other considerations must account for the coupling at the oceans surface with the atmosphere. The evolution occurs over a cylindrical domain $\mathcal{M}=\mathcal{M}_{0} \times(-h, 0)$, where $\mathcal{M}_{0}$ is an open bounded subset of $\mathbb{R}^{2}$ with smooth boundary $\partial \mathcal{M}_{0}$. We denote by $\mathbf{n}_{H}$ the outward unit normal to $\partial \mathcal{M}_{0}$ The boundary $\partial \mathcal{M}$ is partitioned into the top $\Gamma_{i}=\mathcal{M}_{0} \times\{0\}$, bottom $\Gamma_{b}=\mathcal{M}_{0} \times\{-h\}$ and sides $\Gamma_{l}=\partial \mathcal{M}_{0} \times(-h, 0)$. We prescribe (see [33], [42])

$$
\begin{gathered}
\nu_{\mathbf{v}} \partial_{z} \mathbf{v}+\alpha_{\mathbf{v}} \mathbf{v}=0, \quad w=0 \quad \nu_{T} \partial_{z} T+\alpha_{T} T=0, \quad \partial_{z} S=0, \quad \text { on } \Gamma_{i}, \\
\mathbf{v}=0, \quad w=0, \quad \partial_{z} T=0, \quad \partial_{z} S=0, \quad \text { on } \Gamma_{b}, \\
\mathbf{v}=0, \quad \partial_{\mathbf{n}_{H}} T=0, \quad \partial_{\mathbf{n}_{H}} S=0 \quad \text { on } \Gamma_{l} .
\end{gathered}
$$

The equations and boundary conditions $(6.1),(6.2),(6.3),(6.4)$ are supplemented by initial conditions for $\mathbf{v}, T$ and $S$, that is

$$
\mathbf{v}=\mathbf{v}_{0}, \quad T=T_{0}, \quad S=S_{0}, \quad \text { at } t=0 .
$$

\footnotetext{
${ }^{3}$ Actually, in contrast to previous cases above, the convergence is more straightforward since in this case we need to consider only one fixed driving brownian motion $W$ throughout.
} 
With the boundary conditions, (6.2), (6.3), (6.4), we may reformulate (6.1) according to

$$
\begin{gathered}
\partial_{t} \mathbf{v}+(\mathbf{v} \cdot \nabla) \mathbf{v}+w(\mathbf{v}) \partial_{z} \mathbf{v}+\frac{1}{\rho_{0}} \nabla p_{s}-g \int_{z}^{0}\left(\beta_{T} \nabla T+\beta_{S} \nabla S\right) d \bar{z} \\
+f \mathbf{k} \times \mathbf{v}-\mu_{\mathbf{v}} \Delta \mathbf{v}-\nu_{\mathbf{v}} \partial_{z z} \mathbf{v}=F_{\mathbf{v}}+\sigma_{\mathbf{v}}(\mathbf{v}, T, S) \dot{W}_{1}, \\
w(\mathbf{v})=\int_{z}^{0} \nabla \cdot \mathbf{v} d \bar{z}, \quad \int_{-h}^{0} \nabla \cdot \mathbf{v} d \bar{z}=0, \\
\partial_{t} T+(\mathbf{v} \cdot \nabla) T+w(\mathbf{v}) \partial_{z} T-\mu_{T} \Delta T-\nu_{T} \partial_{z z} T=F_{T}+\sigma_{T}(\mathbf{v}, T, S) \dot{W}_{2}, \\
\partial_{t} S+(\mathbf{v} \cdot \nabla) S+w(\mathbf{v}) \partial_{z} S-\mu_{S} \Delta S-\nu_{S} \partial_{z z} S=F_{S}+\sigma_{S}(\mathbf{v}, T, S) \dot{W}_{3},
\end{gathered}
$$

which is the basis for the functional framework that we next recall. Our presentation and notations closely follow the recent survey [42] which covers the deterministic setting.

We will denote by $U$ the triple of prognostic variables, $U=(\mathbf{v}, T, S)$ (comprising four scalar variables), and we set

$$
H=\left\{(\mathbf{v}, T, S) \in L^{2}(\mathcal{M})^{4}: \nabla \cdot \int_{-h}^{0} \mathbf{v} d z=0 \text { over } \mathcal{M}_{0}, \mathbf{n}_{H} \cdot \int_{-h}^{0} \mathbf{v} d z=0 \text { over } \partial \mathcal{M}_{0}, \int_{\mathcal{M}} S d \mathcal{M}=0\right\}
$$

which we equip with the classical $L^{2}$ inner product ${ }^{4}$. Define $P_{H}$ to be the Leray type projection operator from $L^{2}(\mathcal{M})^{4}$ onto $H$. For $H^{1}(\mathcal{M})^{4}$ we consider the subspace:

$$
V=\left\{(\mathbf{v}, T, S) \in H^{1}(\mathcal{M})^{4}: \nabla \cdot \int_{-h}^{0} \mathbf{v} d z=0 \text { over } \mathcal{M}_{0}, \mathbf{v}=0 \text { on } \Gamma_{l} \cup \Gamma_{b}, \int_{\mathcal{M}} S d \mathcal{M}=0\right\}
$$

We equip $V$ with the inner product

$$
\begin{aligned}
\left(\left(U, U^{\sharp}\right)\right) & :=\left(\left(\mathbf{v}, \mathbf{v}^{\sharp}\right)\right)_{1}+\left(\left(T, T^{\sharp}\right)\right)_{2}+\left(\left(S, S^{\sharp}\right)\right)_{3}, \\
((\mathbf{v}, \mathbf{v}))_{1} & :=\int_{\mathcal{M}}\left(\mu_{\mathbf{v}} \nabla \mathbf{v} \cdot \nabla \mathbf{v}^{\sharp}+\nu_{\mathbf{v}} \partial_{z} \mathbf{v} \cdot \partial_{z} \mathbf{v}^{\sharp}\right) d \mathcal{M}+\alpha_{\mathbf{v}} \int_{\Gamma_{i}} \mathbf{v} \mathbf{v}^{\sharp} d \Gamma_{i}, \\
\left(\left(T, T^{\sharp}\right)\right)_{2} & :=\int_{\mathcal{M}}\left(\mu_{T} \nabla T \cdot \nabla T^{\sharp}+\nu_{T} \partial_{z} T \cdot \partial_{z} T^{\sharp}\right) d \mathcal{M}+\alpha_{T} \int_{\Gamma_{i}} T T d \Gamma_{i}, \\
\left(\left(S, S^{\sharp}\right)\right)_{3} & :=\int_{\mathcal{M}}\left(\mu_{S} \nabla S \cdot \nabla S^{\sharp}+\nu_{S} \partial_{z} S \cdot \partial_{z} S^{\sharp}\right) d \mathcal{M} .
\end{aligned}
$$

Note that under these definitions a Poincaré type inequality $|U| \leq C\|U\|$ holds for all $U \in V$. We take $V_{(2)}$ to be the closure of $V$ in the $H^{2}(\mathcal{M})^{4}$ norm and equip this space with the classical $H^{2}(\mathcal{M})$ norm and inner product.

The main linear portion of the equation is defined by ${ }^{5}$

$$
A U=P_{H}\left(\begin{array}{c}
-\mu_{\mathbf{v}} \Delta \mathbf{v}-\nu_{\mathbf{v}} \partial_{z z} \mathbf{v} \\
-\mu_{T} \Delta T-\nu_{T} \partial_{z z} T \\
-\mu_{S} \Delta S-\nu_{S} \partial_{z z} S
\end{array}\right), \quad U=(\mathbf{v}, T, S) \in D(A) .
$$

where we take:

$$
\begin{aligned}
D(A)=\left\{U=(\mathbf{v}, T) \in V_{(2)}:\right. & \nu_{\mathbf{v}} \partial_{z} \mathbf{v}+\alpha_{\mathbf{v}} \mathbf{v}=0, \nu_{T} \partial_{z} T+\alpha_{T} T=0, \partial_{z} S=0 \text { on } \Gamma_{i}, \\
& \left.\partial_{\mathbf{n}_{H}} T=\partial_{\mathbf{n}_{H}} S=0 \text { on } \Gamma_{l}, \partial_{z} T=\partial_{z} S=0 \text { on } \Gamma_{b}\right\} .
\end{aligned}
$$

\footnotetext{
${ }^{4}$ One sometimes also finds the more general definition $\left(U, U^{\sharp}\right):=\int_{\mathcal{M}}\left(\mathbf{v} \cdot \mathbf{v}^{\sharp} d+\kappa_{T} T T^{\sharp}+\kappa_{S} S S^{\sharp}\right) d \mathcal{M}$ with $\kappa_{T}, \kappa_{S}>0$ fixed constants. These parameters $\kappa_{T}, \kappa_{S}$ are useful for the coherence of physical dimensions and for (mathematical) coercivity. Since this is not needed here we take $\kappa_{T}=\kappa_{S}=1$. Similar remarks also apply to the space $V$.

${ }^{5}$ In [42] a slightly different non-selfadjoint operator also called $A$ is given which corresponds to $A+A_{p}(\mathrm{cf}$. (6.8)) in the present manuscript.
} 
We observe that $A$ satisfies the conditions given in Section 2.1. Note also that due to [49] (see also [42]) $|A U| \cong|U|_{H^{2}}$.

We next turn to the quadratically nonlinear terms appearing in (6.6). Noting that there is no momentum equation for $w$ in (6.6) and in accordance with (6.6b) we define the diagnostic function:

$$
w(U)=w(\mathbf{v})=\int_{z}^{0} \nabla \cdot \mathbf{v} d \bar{z}, \quad U=(\mathbf{v}, T, S) \in V .
$$

Take, for $U, U^{\sharp} \in D(A)$ :

$$
B_{1}\left(U, U^{\sharp}\right):=P_{H}\left(\begin{array}{c}
(\mathbf{v} \cdot \nabla) \mathbf{v}^{\sharp} \\
(\mathbf{v} \cdot \nabla) T^{\sharp} \\
(\mathbf{v} \cdot \nabla) S^{\sharp}
\end{array}\right), \quad B_{2}\left(U, U^{\sharp}\right):=P_{H}\left(\begin{array}{c}
w(\mathbf{v}) \partial_{z} \mathbf{v}^{\sharp} \\
w(\mathbf{v}) \partial_{z} T^{\sharp} \\
w(\mathbf{v}) \partial_{z} S^{\sharp}
\end{array}\right)
$$

and let $B\left(U, U^{\sharp}\right):=B\left(U, U^{\sharp}\right)+B\left(U, U^{\sharp}\right)$. As in [42] one may show that $B$ is well defined as an element in $H$ for any $U, U^{\sharp} \in D(A)$. Furthermore $B$ satisfies the conditions (2.3), (2.4), (2.5) relative to the definitions of $A, D(A), V$ and $H$ given here. For the second component of the pressure in (6.6a) we take

$$
A_{p} U=P_{H}\left(\begin{array}{c}
-g \int_{z}^{0}\left(\beta_{T} \nabla T+\beta_{S} \nabla S\right) d \bar{z} \\
0 \\
0
\end{array}\right), \quad U \in V .
$$

and capture the Coriolis forcing in

$$
E U=P_{H}\left(\begin{array}{c}
f \mathbf{k} \times v \\
0 \\
0
\end{array}\right), \quad U \in H
$$

Finally we set

$$
F_{U}=P_{H}\left(\begin{array}{c}
F_{\mathbf{v}} \\
F_{T} \\
F_{S}
\end{array}\right)
$$

We may therefore define

$$
F(U)=A_{p} U+E U+F_{U}
$$

and observe that $F: V \rightarrow H$ and satisfies the requirement (2.8). Finally we define

$$
\sigma((\mathbf{v}, T, S))=\sigma(U)=P_{H}\left(\begin{array}{c}
\sigma_{\mathbf{v}}(U) \\
\sigma_{T}(U) \\
\sigma_{S}(U)
\end{array}\right), \quad U \in H
$$

and assume either (2.10) or (2.11) for the consideration of Martingale or Pathwise solutions receptively.

With the above definitions in place we may write (6.1) supplemented by the boundary conditions (6.2), (6.3), (6.4) in the abstract form (1.1) and conclude via Theorem 2.1:

Theorem 6.1. Assume that $F_{\mathbf{v}}, F_{T}$ and $F_{S}$ are in $L_{l o c}^{2}\left([0, \infty), L^{2}(\mathcal{M})\right)$ and suppose that $\sigma(\cdot)$ associated to $\sigma_{\mathbf{v}}(\cdot), \sigma_{T}(\cdot), \sigma_{S}(\cdot)$ via $(6.12)$ satisfies $(2.10)$. Finally suppose that $\left(\mathbf{v}_{0}, T_{0}, S_{0}\right)$ takes values in $V$ and that $\mu_{0}(\cdot)=\mathbb{P}((\mathbf{v}, T, S) \in \cdot)$ satisfies the moment condition $(2.16)$ with $q \geq 8$. Then:

(i) There exists a local martingale solution of (6.1), the primitive equations of the ocean.

(ii) If we additionally assume (2.11) for $\sigma$ and allow of the relaxation of (2.16) to cover any $q \geq 2$ then there exists a unique maximal, pathwise solution of (6.1). 
Remark 6.1. As noted in the introduction the Stochastic Navier-Stokes equations have been extensively studied. Initially these equations were considered with an additive noise. See [3] and later, [12, 35]. In this case, a classical transformation, allows one to treat $\omega \in \Omega$ as a parameter in the problem. For nonlinear multiplicative noise, the Navier-Stokes equations were initially studied in the context of Martingale solutions. See, for example, [48, 9, 8, 16, 37]. These works typically considered, from the PDE point of view, weak solutions evolving in time only in $L_{x}^{2}$. More recently, pathwise solutions (both local and global in time) for a multiplicative noise have been investigated. See [5, 2, 6, 36]. None of these works addressed the existence and uniqueness of a local pathwise solution in $3 D$ evolving in $H_{x}^{1}$. This case, whose deterministic analogue would be considered classical, was established only recently in [23]. As in the present work a key difficulty involves finding suitable compactness methods to pass to the limit. In [23] the necessary compactness is established by directly showing that the sequence of Galerkin solutions are Cauchy. For an application of this approach to the 2D Primitive equations see [24, 25]. In any case the work here provides an alternative proof of the results in [23]. The reader may readily check that the abstract framework developed in Section 2 applies to the Navier-Stokes equations on a bounded domain with Dirichlet boundary conditions and with $H$ and $V$ corresponding, approximately speaking, to $L_{x}^{2}$ and $H_{x}^{1}$.

\section{The Passage to the Limit}

In this section we provide the details of the passage to the limit, which is used in the proof of the existence of both martingale solutions and pathwise solutions. See Proposition 4.1 and Section 5.2 above.

Proposition 7.1. Let $Z_{k}=\left(\tilde{U}^{m_{k}}, \tilde{W}^{m_{k}}\right)$ be a sequence of $\mathcal{X}$ valued random elements mapping from a probability space $(\tilde{\Omega}, \tilde{\mathcal{F}}, \tilde{\mathbb{P}})$. We assume that

(i) $Z_{k}$ converges almost surely to an element $Z$ in the topology of $\mathcal{X}$, i.e.

$$
\begin{gathered}
\tilde{U}^{m_{k}} \rightarrow \tilde{U} \quad \text { in } L^{2}([0, T], V) \cap C\left([0, T] ; V^{\prime}\right), \\
\tilde{W}^{m_{k}} \rightarrow \tilde{W} \quad \text { in } C\left([0, T] ; \mathfrak{U}_{0}\right) .
\end{gathered}
$$

(ii) Each $\tilde{W}^{m_{k}}$ is a cylindrical Wiener process relative to a filtration $\mathcal{F}_{t}^{m_{k}}$ that contains $\sigma\left(\left(\tilde{W}^{m_{k}}(s), \tilde{U}^{m_{k}}(s)\right)\right.$; $s \leq t)$.

(iii) Each pair $\left(\tilde{U}^{n_{k}}, \tilde{W}^{n_{k}}\right)$ satisfies

$$
\begin{gathered}
d \tilde{U}^{n_{k}}+\left[A \tilde{U}^{n_{k}}+\theta\left(\left\|\tilde{U}^{n_{k}}-\tilde{U}_{*}^{n_{k}}\right\|\right) B^{n_{k}}\left(\tilde{U}^{n_{k}}\right)+F^{n_{k}}\left(\tilde{U}^{n_{k}}\right)\right] d t=\sigma^{n_{k}}\left(\tilde{U}^{n_{k}}\right) d \tilde{W}^{n_{k}}, \\
\tilde{U}^{n_{k}}(0)=P_{n_{k}} \tilde{U}_{0}:=\tilde{U}_{0}^{n_{k}}
\end{gathered}
$$

where we define $\tilde{U}_{*}^{n_{k}}$ by

$$
\frac{d}{d t} \tilde{U}_{*}^{n_{k}}+A \tilde{U}_{*}^{n_{k}}=0 \quad \tilde{U}_{*}^{n_{k}}(0)=\tilde{U}_{0}^{n_{k}}
$$

and assume, for some $p>4$, that

$$
\mathbb{E}\left\|\tilde{U}_{0}\right\|^{p}<\infty
$$

Let $\tilde{\mathcal{S}}=\left(\tilde{\Omega}, \tilde{\mathcal{F}},\left\{\tilde{\mathcal{F}}_{t}\right\}_{t \geq 0}, \tilde{\mathbb{P}}, \tilde{W}\right)$, defining $\tilde{\mathcal{F}}_{t}$ as the completion of $\sigma(\tilde{W}(s), \tilde{U}(s) ; s \leq t)$. Then $(\tilde{\mathcal{S}}, \tilde{U})$ is a global martingale solution of (2.22). Moreover if we define the stopping time:

$$
\tau:=\inf _{t \geq 0}\left\{\left\|\tilde{U}-\tilde{U}_{*}\right\| \geq \kappa\right\}
$$

where $\kappa$ is constant appearing in the definition of $\theta,(3.1)$, then $(\tilde{\mathcal{S}}, \tilde{U}, \tilde{\tau})$ is a local martingale solution of (1.1). 
The rest of this section is devoted to the proof which proceeds in stages. The first step is to establish that the candidate solution is in better spaces via (3.8) and weak compactness arguments. We next establish the almost sure limits of each terms arising in (7.2) against sufficiently 'smooth' test functions $U^{\sharp} \in D(A)$. We then show, using (3.8) for $\tilde{U}^{n_{k}}$ and the Vitali convergence theorem (see e.g. [20]) that each of the deterministic terms converges in $L^{2}(\Omega \times[0, T])$. The convergence of the stochastic terms in (7.2) are facilitated by Lemma 2.1. With these convergences in hand we make use of a variational argument (se e.g. [23]) to finally conclude (2.22) for almost every time $t$ and $\omega \in \tilde{\Omega}$ and with the equality understood in $H$. We pass to the limit for every $t$ by establishing the improved continuity of $U$. See Subsection 7.3 below. This improved continuity justifies the definition of the stopping time $\tau$ specified by (7.5). We therefore infer that for every $t \geq 0$

$$
\int_{0}^{t \wedge \tau} \theta\left(\left\|\tilde{U}-\tilde{U}_{*}\right\|\right) B(\tilde{U}) d s=\int_{0}^{t \wedge \tau} B(\tilde{U}) d s .
$$

In this manner we finally conclude that $(\tilde{S}, \tilde{U}, \tau)$ is a local martingale solution of (1.1) and complete the proof.

\subsection{Improved Regularity of the Candidate Solution}

By applying the Banach - Alaoglu theorem with (3.8) for $\tilde{U}^{n_{k}}$ in the case $p=2$ we infer the existence of elements $\hat{\tilde{U}} \in L^{2}\left(\tilde{\Omega} ; L^{2}([0, T], D(A))\right.$ and $\hat{\tilde{U}} \in L^{2}\left(\tilde{\Omega} ; L^{\infty}([0, T], V)\right)$ such that

$$
\tilde{U}^{n_{k}} \rightarrow \hat{\tilde{U}} \quad \text { in } L^{2}\left(\tilde{\Omega} ; L^{2}([0, T], D(A)),\right.
$$

and

$$
\tilde{U}^{n_{k}} \rightarrow^{*} \hat{\hat{\tilde{U}}} \quad \text { in } L^{2}\left(\tilde{\Omega} ; L^{\infty}([0, T], V) .\right.
$$

On the other hand, due to (7.4), applied to Lemma 3.1, (3.8) we infer that for some $q>2$,

$$
\sup _{k} \mathbb{E} \sup _{t \in[0, T]}\left|\tilde{U}^{n_{k}}\right|_{V^{\prime}}^{q} \leq c \sup _{k} \mathbb{E} \sup _{t \in[0, T]}\left\|\tilde{U}^{n_{k}}\right\|^{q}<\infty .
$$

Thus, with (7.1a), the Vitali convergence theorem implies that,

$$
\tilde{U}^{n_{k}} \rightarrow \tilde{U} \quad \text { in } L^{2}\left(\tilde{\Omega}, L^{\infty}\left(0, T ; V^{\prime}\right)\right) .
$$

Take $\mathcal{R} \subset[0, T] \times \Omega$, measurable and $U^{\sharp} \in D(A)$. By applying (7.7), (7.8), (7.10), we find

$$
\mathbb{E} \int_{0}^{T} \chi_{R}\left\langle\tilde{U}, U^{\sharp}\right\rangle d s=\mathbb{E} \int_{0}^{T} \chi_{R}\left\langle\hat{\tilde{U}}, U^{\sharp}\right\rangle d s=\mathbb{E} \int_{0}^{T} \chi_{R}\left\langle\hat{\tilde{U}}, U^{\sharp}\right\rangle d s,
$$

which means that $\tilde{U}=\hat{\tilde{U}}=\hat{\tilde{U}}$ and we conclude that

$$
\tilde{U} \in L^{2}\left(\tilde{\Omega}, L^{2}([0, T], D(A))\right) \cap L^{2}\left(\tilde{\Omega}, L^{\infty}([0, T], V)\right) .
$$

Furthermore with (7.7) we have

$$
\tilde{U}^{n_{k}} \rightarrow \tilde{U} \quad \text { in } L^{2}\left(\tilde{\Omega} ; L^{2}([0, T], D(A)) .\right.
$$

\subsection{Variational Equality for the Cutoff System}

Fix $U^{\sharp} \in D(A)$. Since, almost surely, $\tilde{U}^{n_{k}} \rightarrow \tilde{U}$ in $L^{2}([0, T], V)$ and noting that

$$
\sup _{k} \mathbb{E}\left[\left(\int_{0}^{T}\left\|\tilde{U}^{n_{k}}\right\|^{2} d t\right)^{2}\right] \leq \sup _{k} c \mathbb{E}\left(\sup _{t \in[0, T]}\left\|\tilde{U}^{n_{k}}\right\|^{4}\right)<\infty
$$


we infer that $\tilde{U}^{n_{k}} \rightarrow \tilde{U}$ in $L^{2}\left(\Omega, L^{2}([0, T], V)\right)$, by the Vitali convergence theorem. By thinning the sequence $n_{k}$ if necessary, we may also conclude that

$$
\left\|\tilde{U}^{n_{k}}-\tilde{U}\right\|^{2} \rightarrow 0
$$

for almost every $(t, \omega) \in[0, T] \times \tilde{\Omega}$.

The pointwise convergence in the linear term is direct:

$$
\left|\int_{0}^{t}\left\langle A\left(\tilde{U}^{n_{k}}-\tilde{U}\right), U^{\sharp}\right\rangle d s\right| \leq c\left\|U^{\sharp}\right\|\left(\int_{0}^{T}\left\|\tilde{U}^{n_{k}}-\tilde{U}\right\|^{2} d s\right)^{1 / 2} .
$$

We conclude that for almost every $(t, \omega) \in[0, T] \times \tilde{\Omega}$

$$
\int_{0}^{t}\left\langle A \tilde{U}^{n_{k}}, U^{\sharp}\right\rangle d s \rightarrow \int_{0}^{t}\left\langle A \tilde{U}, U^{\sharp}\right\rangle d s .
$$

For $B$ we estimate

$$
\begin{aligned}
& \left|\int_{0}^{t}\left\langle\theta\left(\left\|\tilde{U}^{n_{k}}-\tilde{U}_{*}^{n_{k}}\right\|\right) B^{n_{k}}\left(\tilde{U}^{n_{k}}\right)-\theta\left(\left\|\tilde{U}-\tilde{U}_{*}\right\|\right) B(\tilde{U}), U^{\sharp}\right\rangle d s\right| \\
& \leq\left|\int_{0}^{t}\left\langle\theta\left(\left\|\tilde{U}^{n_{k}}-\tilde{U}_{*}^{n_{k}}\right\|\right)\left(B^{n_{k}}\left(\tilde{U}^{n_{k}}\right)-B(\tilde{U})\right), U^{\sharp}\right\rangle d s\right|+\left|\int_{0}^{t}\left\langle\left(\theta\left(\left\|\tilde{U}^{n_{k}}-\tilde{U}_{*}^{n_{k}}\right\|\right)-\theta\left(\left\|\tilde{U}-\tilde{U}_{*}\right\|\right)\right) B(\tilde{U}), U^{\sharp}\right\rangle d s\right| \\
& \leq\left|\int_{0}^{t}\left\langle\theta\left(\left\|\tilde{U}^{n_{k}}-\tilde{U}_{*}^{n_{k}}\right\|\right)\left(B\left(\tilde{U}^{n_{k}}\right)-B(\tilde{U})\right), P_{n_{k}} U^{\sharp}\right\rangle d s\right|+\left|\int_{0}^{t}\left\langle\theta\left(\left\|\tilde{U}^{n_{k}}-\tilde{U}_{*}^{n_{k}}\right\|\right) B(\tilde{U}), Q_{n_{k}} U^{\sharp}\right\rangle d s\right| \\
& \quad+\left|\int_{0}^{t}\left\langle\left(\theta\left(\left\|\tilde{U}^{n_{k}}-\tilde{U}_{*}^{n_{k}}\right\|\right)-\theta\left(\left\|\tilde{U}-\tilde{U}_{*}\right\|\right)\right) B(\tilde{U}), U^{\sharp}\right\rangle d s\right| \\
& :=J_{1}^{n_{k}}+J_{2}^{n_{k}}+J_{3}^{n_{k}} .
\end{aligned}
$$

We address the elements on the right hand side in reverse order. Due to (7.14) we have, for almost every $(t, \omega) \in[0, T] \times \tilde{\Omega}:^{6}$

$$
\left\|\tilde{U}^{n}-\tilde{U}_{*}^{n}\right\| \rightarrow\left\|\tilde{U}-\tilde{U}_{*}\right\| .
$$

We therefore infer,

$$
\theta\left(\left\|\tilde{U}^{n}-\tilde{U}_{*}^{n}\right\|\right) \rightarrow \theta\left(\left\|\tilde{U}-\tilde{U}_{*}\right\|\right),
$$

almost everywhere on $[0, T] \times \tilde{\Omega}$. By assumptions (2.3), (2.4),

$$
\left|\left\langle\left(\theta\left(\left\|\tilde{U}^{n_{k}}-\tilde{U}_{*}^{n_{k}}\right\|\right)-\theta\left(\left\|\tilde{U}-\tilde{U}_{*}\right\|\right)\right) B(\tilde{U}), U^{\sharp}\right\rangle\right| d t \leq c\left|A U^{\sharp}\right|\|\tilde{U}\|^{2},
$$

and since,

$$
\begin{aligned}
\mathbb{E} \int_{0}^{T} \int_{0}^{t} & \left\langle\left\langle\left(\theta\left(\left\|\tilde{U}^{n_{k}}-\tilde{U}_{*}^{n_{k}}\right\|\right)-\theta\left(\left\|\tilde{U}-\tilde{U}_{*}\right\|\right)\right) B(\tilde{U}), U^{\sharp}\right\rangle\right| d s d t \\
& \leq c \mathbb{E} \int_{0}^{T}\left|\left\langle\left(\theta\left(\left\|\tilde{U}^{n_{k}}-\tilde{U}_{*}^{n_{k}}\right\|\right)-\theta\left(\left\|\tilde{U}-\tilde{U}_{*}\right\|\right)\right) B(\tilde{U}), U^{\sharp}\right\rangle\right| d t,
\end{aligned}
$$

the Lebesgue dominated convergence theorem therefore implies:

$$
\mathbb{E} \int_{0}^{T} J_{3}^{n_{k}} d t \rightarrow 0 .
$$

\footnotetext{
${ }^{6}$ Since $\tilde{U}_{0}^{n} \rightarrow \tilde{U}_{0}$ in $L^{2}(\tilde{\Omega} ; V)$, it follows that $\mathbb{E}\left(\sup _{t \in[0, T]}\left\|\tilde{U}_{*}^{n}-\tilde{U}_{*}\right\|^{2}\right) \rightarrow 0$.
} 
Local Martingale and Pathwise Solutions for an Abstract Fluids Model

Thinning the sequence, if necessary we conclude that

$$
J_{3}^{n_{k}} \rightarrow 0 \text { a.e. }(t, \omega) \in[0, T] \times \tilde{\Omega} .
$$

We estimate the second term, $J_{2}^{n_{k}}$ according to

$$
J_{2}^{n_{k}} \leq\left|Q_{n_{k}} A U^{\sharp}\right| \int_{0}^{T}\|\tilde{U}\|^{2} d s \rightarrow 0,
$$

for almost every $(t, \omega)$. Finally for $J_{1}^{n_{k}}$, the bilinearity of $B$ implies that

$$
B\left(\tilde{U}^{n_{k}}, \tilde{U}^{n_{k}}\right)-B(\tilde{U}, \tilde{U})=B\left(\tilde{U}^{n_{k}}-\tilde{U}, \tilde{U}^{n_{k}}\right)+B\left(\tilde{U}, \tilde{U}^{n_{k}}-\tilde{U}\right) .
$$

Again by the assumptions (2.3), (2.4) we infer,

$$
\begin{aligned}
J_{1}^{n_{k}} & \leq c\left|A U^{\sharp}\right| \int_{0}^{T}\left(\left\|\tilde{U}^{n_{k}}\right\|+\|\tilde{U}\|\right)\left\|\tilde{U}^{n_{k}}-\tilde{U}\right\| d s \\
& \leq c\left|A U^{\sharp}\right|\left(\int_{0}^{T}\left(\left\|\tilde{U}^{n_{k}}\right\|^{2}+\|\tilde{U}\|^{2}\right) d s\right)^{1 / 2}\left(\int_{0}^{T}\left\|\tilde{U}^{n_{k}}-\tilde{U}\right\|^{2} d s\right)^{1 / 2} .
\end{aligned}
$$

Thus, with assumption (7.1a), we infer that

$$
J_{1}^{n_{k}} \rightarrow 0 \quad \text { for almost all }(t, \omega) .
$$

Combining (7.18), (7.17), (7.16) we conclude that, for almost every $(t, \omega)$,

$$
\int_{0}^{t}\left\langle\theta\left(\left\|\tilde{U}^{n_{k}}-\tilde{U}_{*}^{n_{k}}\right\|\right) B^{n}\left(\tilde{U}^{n}\right), U^{\sharp}\right\rangle d s \rightarrow \int_{0}^{t}\left\langle\theta\left(\left\|\tilde{U}-\tilde{U}_{*}\right\|\right) B(\tilde{U}), U^{\sharp}\right\rangle d s .
$$

For the remaining deterministic terms we estimate

$$
\left|\int_{0}^{t}\left\langle F^{n_{k}}\left(\tilde{U}^{n_{k}}\right)-F(\tilde{U}), U^{\sharp}\right\rangle d s\right| \leq c\left|U^{\sharp}\right| \int_{0}^{t}\left|F\left(\tilde{U}^{n_{k}}\right)-F(\tilde{U})\right| d s+\left|Q_{n_{k}} U^{\sharp}\right| \int_{0}^{t}|F(\tilde{U})| d s:=J_{4}^{n_{k}}+J_{5}^{n_{k}} .
$$

Due to (7.14) and the continuity assumed for $F,(2.7)$, we infer that for almost every $(\omega, t)$,

$$
\left|F\left(\tilde{U}^{n_{k}}\right)-F(\tilde{U})\right| \rightarrow 0 \text {. }
$$

On the other hand by (2.7),

$$
\left|F\left(\tilde{U}^{n_{k}}\right)-F(\tilde{U})\right| \leq c\left(1+\left\|\tilde{U}^{n_{k}}\right\|+\|\tilde{U}\|\right),
$$

and we infer that,

$$
\sup _{k} \mathbb{E} \int_{0}^{T}\left|F\left(\tilde{U}^{n_{k}}\right)-F(\tilde{U})\right|^{2} d t \leq c \sup _{k} \mathbb{E} \int_{0}^{T}\left(1+\left\|\tilde{U}^{n_{k}}\right\|^{2}+\|\tilde{U}\|^{2}\right) d t<\infty .
$$

In consequence $\left\{\left|F\left(\tilde{U}^{n_{k}}\right)-F(\tilde{U})\right|\right\}_{k \geq 0}$ is uniformly integrable over $\tilde{\Omega} \times[0, T]$. By applying the Vitali Convergence Theorem we have $\mathbb{E} \int_{0}^{T}\left|F^{n_{k}}\left(\tilde{U}^{n_{k}}\right)-F(\tilde{U})\right| d t \rightarrow 0$. Thinning the sequence further if needed, we infer that almost everywhere in $\tilde{\Omega}$.

$$
\int_{0}^{t}\left|F^{n_{k}}\left(\tilde{U}^{n_{k}}\right)-F(\tilde{U})\right| d t \leq \int_{0}^{T}\left|F^{n_{k}}\left(\tilde{U}^{n_{k}}\right)-F(\tilde{U})\right| d t \rightarrow 0 .
$$

in order to finally conclude that

$$
J_{4}^{n_{k}} \rightarrow 0 \text { a.e. }(\omega, t) .
$$


Turning to the second term $J_{5}^{n_{k}}$, we see, again as a consequence of the assumption (2.7), that $\int_{0}^{t}|F(\tilde{U})| d s \leq$ $c \int_{0}^{T}(1+\|\tilde{U}\|) d s<\infty$ and so

$$
J_{5}^{n_{k}} \rightarrow 0 \quad \text { a.e. }(\omega, t) .
$$

In conclusion, by (7.21), (7.22) we finally have

$$
\int_{0}^{t}\left\langle F^{n_{k}}\left(\tilde{U}^{n_{k}}\right), U^{\sharp}\right\rangle d s \rightarrow \int_{0}^{t}\left\langle F(\tilde{U}), U^{\sharp}\right\rangle d s
$$

for almost every $(\omega, t) \in \tilde{\Omega} \times[0, T]$.

We next establish the convergences to the deterministic terms in $(2.22)$ in the space $L^{q}(\tilde{\Omega} \times[0, T])$, $1 \leq q<2$. Notice that due to $(2.4),(2.7)$,

$$
\begin{aligned}
& \mathbb{E} \int_{0}^{T}\left|\int_{0}^{t}\left\langle A \tilde{U}^{n_{k}}+\theta\left(\left\|\tilde{U}^{n_{k}}-\tilde{U}_{*}^{n_{k}}\right\|\right) B^{n}\left(\tilde{U}^{n_{k}}\right)+F^{n}\left(\tilde{U}^{n_{k}}\right), U^{\sharp}\right\rangle d s\right|^{2} d t \\
& \quad \leq c \mathbb{E} \int_{0}^{T}\left|\left\langle A \tilde{U}^{n_{k}}+\theta\left(\left\|\tilde{U}^{n_{k}}-\tilde{U}_{*}^{n_{k}}\right\|\right) B^{n}\left(\tilde{U}^{n_{k}}\right)+F^{n}\left(\tilde{U}^{n_{k}}\right), U^{\sharp}\right\rangle\right|^{2} d s \\
& \quad \leq c\left|A U^{\sharp}\right|^{2} \mathbb{E} \int_{0}^{T}\left(\left\|\tilde{U}^{n_{k}}\right\|^{2}+\left\|\tilde{U}^{n_{k}}\right\|^{4}+1\right) d s .
\end{aligned}
$$

Thus, for every $q \in[1,2)$,

$$
\left\{\int_{0}^{t}\left\langle A \tilde{U}^{n_{k}}+\theta\left(\left\|\tilde{U}^{n_{k}}-\tilde{U}_{*}^{n_{k}}\right\|\right) B^{n_{k}}\left(\tilde{U}^{n_{k}}\right)+F^{n_{k}}\left(\tilde{U}^{n_{k}}\right), U^{\sharp}\right\rangle d s\right\}_{k \geq 0}
$$

is uniformly integrable in $L^{q}(\tilde{\Omega} \times[0, T])$.

Combining this with (7.15), (7.19), (7.23) we conclude that for every $q \in[1,2)$ :

$$
\begin{gathered}
\int_{0}^{t}\left\langle A \tilde{U}^{n_{k}}+\theta\left(\left\|\tilde{U}^{n_{k}}-\tilde{U}_{*}^{n_{k}}\right\|\right) B^{n_{k}}\left(\tilde{U}^{n_{k}}\right)+F^{n_{k}}\left(\tilde{U}^{n_{k}}\right), U^{\sharp}\right\rangle d s \\
\longrightarrow \int_{0}^{t}\left\langle A \tilde{U}+\theta\left(\left\|\tilde{U}-\tilde{U}_{*}\right\|\right) B(\tilde{U})+F(\tilde{U}), U^{\sharp}\right\rangle d s,
\end{gathered}
$$

in $L^{q}([0, T] \times \Omega)$.

The stochastic terms are handled differently. Using (2.2) and (2.10) we estimate

$$
\begin{aligned}
\left\|\sigma^{n_{k}}\left(\tilde{U}^{n_{k}}\right)-\sigma(\tilde{U})\right\|_{L_{2}(\mathfrak{U}, H)} & \leq\left\|\sigma\left(\tilde{U}^{n_{k}}\right)-\sigma(\tilde{U})\right\|_{L_{2}(\mathfrak{U}, H)}+\left\|Q_{n_{k}} \sigma(\tilde{U})\right\|_{L_{2}(\mathfrak{U}, H)} \\
& \leq\left\|\sigma\left(\tilde{U}^{n_{k}}\right)-\sigma(\tilde{U})\right\|_{L_{2}(\mathfrak{U}, H)}+\frac{1}{\lambda_{n_{k}}^{1 / 2}}\|\sigma(\tilde{U})\|_{L_{2}(\mathfrak{U}, V)} \\
& \leq\left\|\sigma\left(\tilde{U}^{n_{k}}\right)-\sigma(\tilde{U})\right\|_{L_{2}(\mathfrak{U}, H)}+\frac{c}{\lambda_{n_{k}}^{1 / 2}}(1+\|\tilde{U}\|) .
\end{aligned}
$$

Thus, due to (7.14) and the assumed continuity of $\sigma$ (see (2.10)) we conclude that

$$
\left\|\sigma^{n_{k}}\left(\tilde{U}^{n_{k}}\right)-\sigma(\tilde{U})\right\|_{L_{2}(\mathfrak{U}, H)} \rightarrow 0,
$$

for almost every $(\omega, t) \in \tilde{\Omega} \times[0, T]$. On the other hand, we observe that

$$
\sup _{n_{k}} \mathbb{E}\left(\int_{0}^{T}\left\|\sigma^{n_{k}}\left(\tilde{U}^{n_{k}}\right)\right\|_{L_{2}(\mathfrak{U}, H)}^{4} d s\right) \leq c \sup _{n_{k}} \mathbb{E}\left(\int_{0}^{T}\left(1+\left\|\tilde{U}^{n_{k}}\right\|^{4}\right) d s\right),
$$


where we have again made use of the sublinear condition (2.10). We therefore infer that $\left\|\sigma^{n_{k}}\left(\tilde{U}^{n_{k}}\right)\right\|_{L_{2}(\mathfrak{U}, H)}$ is uniformly integrable in $L^{p}(\Omega \times[0, T])$ for any $p \in[1,4)$. With the Vitali convergence theorem we infer, for all such $p \in[1,4)$,

$$
\sigma^{n_{k}}\left(\tilde{U}^{n_{k}}\right) \rightarrow \sigma(\tilde{U}) \quad \text { in } L^{p}\left(\tilde{\Omega} ; L^{p}\left([0, T], L_{2}(\mathfrak{U}, H)\right)\right) .
$$

In particular (7.25) implies the convergence in probability of $\sigma^{n_{k}}\left(\tilde{U}^{n_{k}}\right)$ in $\left.L^{2}\left([0, T], L_{2}(\mathfrak{U}, H)\right)\right)$. Thus, along with the assumption (7.1b), we apply Lemma 2.15 and infer that

$$
\int_{0}^{t} \sigma^{n_{k}}\left(\tilde{U}^{n_{k}}\right) d \tilde{W}_{n_{k}} \rightarrow \int_{0}^{t} \sigma(\tilde{U}) d \tilde{W}
$$

in probability $\left.L^{2}([0, T], H)\right)$. Another application of the Vitali convergence theorem using estimates involving (2.12), (7.25) shows that the convergence in (7.26) occurs moreover in $L^{2}\left(\Omega ; L^{2}([0, T], H)\right)$.

With the above details in hand we now establish $(2.22)$ in a variational sense. Fix any $U^{\sharp} \in D(A)$, $\mathcal{R} \subset \tilde{\Omega} \times[0, T]$ measurable. Using (7.13) and then (7.24) and (7.26) we observe that

$$
\begin{aligned}
\mathbb{E} & \int_{0}^{T} \chi_{\mathcal{R}}\left\langle\tilde{U}, U^{\sharp}\right\rangle d t=\lim _{k \rightarrow \infty} \mathbb{E} \int_{0}^{T} \chi_{\mathcal{R}}\left\langle\tilde{U}^{n_{k}}, U^{\sharp}\right\rangle d t \\
= & \lim _{k \rightarrow \infty} \mathbb{E} \int_{0}^{T} \chi_{\mathcal{R}}\left\langle\tilde{U}_{0}^{n_{k}}, U^{\sharp}\right\rangle d t \\
& -\lim _{k \rightarrow \infty} \mathbb{E} \int_{0}^{T} \chi_{\mathcal{R}}\left(\int_{0}^{t}\left\langle A \tilde{U}^{n_{k}}, U^{\sharp}\right\rangle d s\right) d t \\
& -\lim _{k \rightarrow \infty} \mathbb{E} \int_{0}^{T} \chi_{\mathcal{R}}\left(\int_{0}^{t}\left\langle\theta\left(\left\|\tilde{U}^{n_{k}}-\tilde{U}_{*}^{n_{k}}\right\|\right) B^{n_{k}}\left(\tilde{U}^{n_{k}}\right), U^{\sharp}\right\rangle d s\right) d t \\
& -\lim _{k \rightarrow \infty} \mathbb{E} \int_{0}^{T} \chi_{\mathcal{R}}\left(\int_{0}^{t}\left\langle F^{n_{k}}\left(\tilde{U}^{n_{k}}\right), U^{\sharp}\right\rangle d s\right) d t \\
& +\lim _{k \rightarrow \infty} \mathbb{E} \int_{0}^{T} \chi_{\mathcal{R}}\left(\int_{0}^{t}\left\langle\sigma^{n_{k}}\left(\tilde{U}^{n_{k}}\right), U^{\sharp}\right\rangle d W^{n_{k}}\right) d t \\
= & \mathbb{E} \int_{0}^{T} \chi_{\mathcal{R}}\left(\left\langle\tilde{U}_{0}, U^{\sharp}\right\rangle-\int_{0}^{t}\left\langle A \tilde{U}+\theta\left(\left\|\tilde{U}-\tilde{U}_{*}\right\|\right) B(\tilde{U})+F(\tilde{U}), U^{\sharp}\right\rangle d s\right) d t \\
& +\mathbb{E} \int_{0}^{T} \chi_{\mathcal{R}}\left(\int_{0}^{t}\left\langle\sigma(\tilde{U}), U^{\sharp}\right\rangle d W\right) d t .
\end{aligned}
$$

Since this equality holds over all such $\mathcal{R}$ we may conclude that for almost every $(\omega, t) \in \tilde{\Omega} \times[0, T]$ and every $U^{\sharp} \in D(A)$ that,

$$
\left\langle\tilde{U}(t), U^{\sharp}\right\rangle+\int_{0}^{t}\left\langle A \tilde{U}+\theta\left(\left\|\tilde{U}-\tilde{U}_{*}\right\|\right) B(\tilde{U})+F(\tilde{U}), U^{\sharp}\right\rangle d s=\left\langle\tilde{U}_{0}, U^{\sharp}\right\rangle+\int_{0}^{t}\left\langle\sigma(\tilde{U}), U^{\sharp}\right\rangle d W .
$$

Moreover, due to (7.12) established above, it follows by density that (7.27) holds also over $U^{\sharp} \in H$ and hence (2.22) in the analogous sense to (2.19).

\subsection{Improved Regularity In Time}

With (7.27) and (7.12) in hand it remains only to establish better continuity, in time, for $U$. More precisely, we must show that $\tilde{U} \in C([0, T] ; V)$ a.s. Of course, such a condition is needed in order to justify the definition (7.5).

To this end we define

$$
d Z+A Z=\sigma(\tilde{U}) d \tilde{W}, \quad Z(0)=\tilde{U}_{0} .
$$


Observe that since $\sigma(\tilde{U}) \in L^{2}\left(\Omega, L^{2}\left([0, T], L_{2}(\mathfrak{U}, V)\right)\right)$ we have

$$
Z \in L^{2}(\tilde{\Omega}, C([0, T], V)) \cap L^{2}\left(\tilde{\Omega}, L^{2}([0, T] ; D(A))\right) .
$$

Now take $\bar{U}=\tilde{U}-Z$. Subtracting (7.28) from (2.22) we find

$$
\begin{gathered}
\frac{d}{d t} \bar{U}+A \bar{U}+\theta\left(\left\|\bar{U}+Z-\tilde{U}_{*}\right\|\right) B(\bar{U}+Z)+F(\bar{U}+Z)=0, \\
\bar{U}(0)=\tilde{U}_{0} .
\end{gathered}
$$

Due to (7.29), (7.12), we infer that $\bar{U} \in L^{2}\left(\tilde{\Omega}, L^{2}(0, T ; D(A)) \cap L^{\infty}([0, T], V)\right.$ and hence that,

$$
A \bar{U}, \quad \theta\left(\left\|\bar{U}+Z-\tilde{U}_{*}\right\|\right) B(\bar{U}+Z), \quad F(\bar{U}+Z) \in L^{2}\left(\tilde{\Omega}, L^{2}([0, T], H) .\right.
$$

We conclude with (7.30) that

$$
\frac{d}{d t} A^{1 / 2} \bar{U} \in L^{2}\left(\Omega ; L^{2}\left(0, T ; V^{\prime}\right)\right), \quad A^{1 / 2} \bar{U} \in L^{2}\left(\Omega ; L^{2}(0, T ; V)\right)
$$

By applying [47, Chapter 3, Lemma 1.2] we infer that $A^{1 / 2} \bar{U} \in C([0, T], H)$ so that, with (7.29), we deduce that

$$
\tilde{U} \in C([0, T] ; V), \text { a.s. }
$$

With (7.27), (7.12), and (7.33) we finally conclude that $(\tilde{\mathcal{S}}, \tilde{U})$ is a global Martingale solution of (2.22). Furthermore, having justified (7.5) and applying (7.6) to $(7.27)$ we have that $(\tilde{\mathcal{S}}, \tilde{U}, \tau)$ is a local Martingale solution of (1.1). The proof of Proposition 7.1 is therefore complete.

\section{Appendix: Proof of the Convergence Theorem}

In this final section we provide a proof of Lemma 2.1. Convergence results similar to Lemma 2.1 have appeared in previous works (see e.g. [1], [28]). However, to the best of our knowledge, no one up to the present has provided a detailed proof. Note that in the present work Lemma 2.1 is an important technical tool for the passage to the limit, as detailed above in Section 7.

To simplify the exposition, we begin by introducing the notations:

$$
\mathcal{I}^{n}:=\int_{0}^{t} G^{n} d W^{n}=\sum_{k \geq 0} \int_{0}^{t} G_{k}^{n} d W_{k}^{n}=\sum_{k \geq 0} Y_{k}^{n}, \quad \mathcal{I}:=\int_{0}^{t} G d W=\sum_{k} \int_{0}^{t} G_{k} d W_{k}=\sum_{k} Y_{k} .
$$

For the truncations we set

$$
\mathcal{I}_{N}^{n}:=\sum_{N \geq k \geq 0} Y_{k}^{n}, \quad \mathcal{J}_{N}^{n}:=\mathcal{I}^{n}-\mathcal{I}_{N}^{n}, \quad \mathcal{I}_{N}:=\sum_{N \geq k \geq 0} Y_{k}, \quad \mathcal{J}_{N}:=\mathcal{I}^{n}-\mathcal{I}_{N}^{n}
$$

With these notations we now split

$$
\left|\mathcal{I}^{n}-\mathcal{I}\right|_{L^{2}([0, T], X)} \leq\left|\mathcal{I}^{n}-\mathcal{I}_{N}^{n}\right|_{L^{2}([0, T], X)}+\left|\mathcal{I}_{N}^{n}-\mathcal{I}_{N}\right|_{L^{2}([0, T], X)}+\left|\mathcal{I}_{N}-\mathcal{I}\right|_{L^{2}([0, T], X)}
$$

and observe that the proof of Lemma 2.1 is complete once we establish that

$$
\left\{\begin{array}{l}
\text { For every } \epsilon>0, \lim _{N \rightarrow \infty} \sup _{n \geq N} \mathbb{P}\left(\left|\mathcal{J}_{N}^{n}\right|_{L^{2}([0, T], X)}>\epsilon\right)=0, \\
\lim _{n \rightarrow \infty}\left|Y_{k}^{n}-Y_{k}\right|_{L^{2}([0, T], X)}=0 \text { in Probability, for each fixed } k, \\
\lim _{N \rightarrow \infty}\left|\mathcal{J}_{N}\right|_{L^{2}([0, T], X)}=0 \text { in Probability. }
\end{array}\right.
$$


Local Martingale and Pathwise Solutions for an Abstract Fluids Model

To establish each of the convergences in (8.1) we make extensive use of the following martingale inequality (see e.g. [21])

$$
\mathbb{P}\left(\int_{0}^{T}\left|\int_{0}^{t} F d W\right|_{X}^{2} d t>c\right) \leq \frac{\kappa T}{c}+\mathbb{P}\left(\int_{0}^{T}|F|_{L_{2}(\mathfrak{U}, X)}^{2} d t>\kappa\right) .
$$

Here $c, \kappa$ may be any positive constants and $F$ any $\mathcal{F}_{t}$ predictable element in $L^{2}\left([0, T] ; L_{2}(\mathfrak{U}, X)\right)$. For the first item in (8.1) we apply (8.2) and observe that for any $\epsilon, \delta>0$

$$
\begin{aligned}
\mathbb{P}\left(\left|\mathcal{J}_{N}^{n}\right|_{L^{2}([0, T], X)}>\epsilon\right) & \leq \frac{\delta}{3}+\mathbb{P}\left(\sum_{k \geq N} \int_{0}^{T}\left|G_{k}^{n}\right|^{2} d t>\frac{\delta \epsilon^{2}}{3 T}\right) \\
& \leq \frac{\delta}{3}+\mathbb{P}\left(\int_{0}^{T}\left|G^{n}-G\right|_{L_{2}(\mathfrak{U}, X)}^{2} d t>\frac{\delta \epsilon^{2}}{12 T}\right)+\mathbb{P}\left(\sum_{k \geq N} \int_{0}^{T}|G|^{2} d t>\frac{\delta \epsilon^{2}}{12 T}\right) .
\end{aligned}
$$

With this estimate, the assumptions on $G$ and (2.14b) we infer the first item in (8.1). The third item in (8.1) is established in similar manner via an application of (8.2).

It remains to address the second item in (8.1). In order to treat these terms we introduce the functional:

$$
\mathcal{R}_{\rho}(F)=\frac{1}{\rho} \int_{0}^{t} \exp \left(-\frac{t-s}{\rho}\right) F(s) d s \quad F \in L^{1}([0, T], X), \rho>0 .
$$

Using this functional and then integrating by parts we estimate

$$
\begin{aligned}
& \left|Y_{k}^{n}-Y_{k}\right|_{X}=\left|\int_{0}^{t} G_{k}^{n} d W_{k}^{n}-\int_{0}^{t} G_{k} d W_{k}\right|_{X} \\
& \leq\left|\int_{0}^{t}\left(G_{k}^{n}-\mathcal{R}_{\rho}\left(G_{k}^{n}\right)\right) d W_{k}^{n}\right|_{X}+\left|\int_{0}^{t}\left(\mathcal{R}_{\rho}\left(G_{k}\right)-G_{k}\right) d W_{k}\right|_{X}+\left|\int_{0}^{t} \mathcal{R}_{\rho}\left(G_{k}^{n}\right) d W_{k}^{n}-\int_{0}^{t} \mathcal{R}_{\rho}\left(G_{k}\right) d W_{k}\right|_{X}^{2} \\
& \leq\left|\int_{0}^{t}\left(G_{k}^{n}-\mathcal{R}_{\rho}\left(G_{k}^{n}\right)\right) d W_{k}^{n}\right|_{X}+\left|\int_{0}^{t}\left(\mathcal{R}_{\rho}\left(G_{k}\right)-G_{k}\right) d W_{k}\right|_{X}+\left|\mathcal{R}_{\rho}\left(G_{k}\right) W_{k}-\mathcal{R}_{\rho}\left(G_{k}^{n}\right) W_{k}^{n}\right|_{X} \\
& \quad+\frac{1}{\rho}\left|\int_{0}^{t}\left(\mathcal{R}_{\rho}\left(G_{k}\right) W_{k}-\mathcal{R}_{\rho}\left(G_{k}^{n}\right) W_{k}^{n}\right) d s\right|_{X}+\frac{1}{\rho}\left|\int_{0}^{t}\left(G_{k} W_{k}-G_{k}^{n} W_{k}^{n}\right) d s\right|_{X} .
\end{aligned}
$$

We now proceed to treat each of the term on the right hand side of (8.4). Fix $\epsilon, \delta>0$. For the first term in (8.4) we apply (8.2) and estimate

$$
\begin{aligned}
& \mathbb{P}\left(\left|\int_{0}^{t}\left(G_{k}^{n}-\mathcal{R}_{\rho}\left(G_{k}^{n}\right)\right) d W_{k}^{n}\right|_{L^{2}([0, T] ; X)}>\epsilon\right) \leq \delta+\mathbb{P}\left(\int_{0}^{T}\left|G_{k}^{n}-\mathcal{R}_{\rho}\left(G_{k}^{n}\right)\right|_{X}^{2} d t>\frac{\delta \epsilon^{2}}{T}\right) \\
& \leq \delta \mathbb{P}\left(\int_{0}^{T}\left|G_{k}^{n}-G_{k}\right|_{X}^{2} d t>\frac{\delta \epsilon^{2}}{3 T}\right)+\mathbb{P}\left(\int_{0}^{T}\left|G_{k}-\mathcal{R}_{\rho}\left(G_{k}\right)\right|_{X}^{2} d t>\frac{\delta \epsilon^{2}}{3 T}\right) \\
&+\mathbb{P}\left(\int_{0}^{T}\left|\mathcal{R}_{\rho}\left(G_{k}\right)-\mathcal{R}_{\rho}\left(G_{k}^{n}\right)\right|_{X}^{2} d t>\frac{\delta \epsilon^{2}}{3 T}\right) \\
& \leq+2 \mathbb{P}\left(\int_{0}^{T}\left|G_{k}^{n}-G_{k}\right|_{X}^{2} d t>\frac{\delta \epsilon^{2}}{3 T}\right)+\mathbb{P}\left(\int_{0}^{T}\left|G_{k}-\mathcal{R}_{\rho}\left(G_{k}\right)\right|_{X}^{2} d t>\frac{\delta \epsilon^{2}}{3 T}\right) .
\end{aligned}
$$

With (8.2) we also find that

$$
\mathbb{P}\left(\left|\int_{0}^{t}\left(\mathcal{R}_{\rho}\left(G_{k}\right)-G_{k}\right) d W_{k}\right|_{L^{2}([0, T] ; X)}>\epsilon\right) \leq \delta+\mathbb{P}\left(\int_{0}^{T}\left|G_{k}-\mathcal{R}_{\rho}\left(G_{k}\right)\right|_{X}^{2} d t>\frac{\delta \epsilon^{2}}{T}\right)
$$


The last three items are treated differently

$$
\begin{aligned}
& \mathbb{P}\left(\left|\mathcal{R}_{\rho}\left(G_{k}\right) W_{k}-\mathcal{R}_{\rho}\left(G_{k}^{n}\right) W_{k}^{n}\right|_{L^{2}([0, T] ; X)}>\epsilon\right) \\
& \leq \mathbb{P}\left(\int_{0}^{T}\left|\mathcal{R}_{\rho}\left(G_{k}\right) W_{k}^{n}-\mathcal{R}_{\rho}\left(G_{k}^{n}\right) W_{k}^{n}\right|_{X}^{2} d t>\frac{\epsilon^{2}}{4}\right)+\mathbb{P}\left(\int_{0}^{T}\left|\mathcal{R}_{\rho}\left(G_{k}\right) W_{k}-\mathcal{R}_{\rho}\left(G_{k}\right) W_{k}^{n}\right|_{X}^{2} d t>\frac{\epsilon^{2}}{4}\right) \\
& \quad \leq \mathbb{P}\left(\sup _{t \in[0, T]}\left|W_{k}^{n}\right|^{2} \int_{0}^{T}\left|G_{k}-G_{k}^{n}\right|_{X}^{2} d t>\frac{\epsilon^{2}}{4}\right)+\mathbb{P}\left(\sup _{t \in[0, T]}\left|W_{k}-W_{k}^{n}\right|^{2} \int_{0}^{T}\left|G_{k}\right|_{X}^{2} d t>\frac{\epsilon^{2}}{4}\right) .
\end{aligned}
$$

Similar estimates lead to

$$
\begin{aligned}
& \mathbb{P}\left(\left|\frac{1}{\rho} \int_{0}^{t}\left(\mathcal{R}_{\rho}\left(G_{k}\right) W_{k}-\mathcal{R}_{\rho}\left(G_{k}^{n}\right) W_{k}^{n}\right) d s\right|_{L^{2}([0, T] ; X)}>\epsilon\right) \\
& \quad \leq \mathbb{P}\left(\sup _{t \in[0, T]}\left|W_{k}^{n}\right|^{2} \int_{0}^{T}\left|G_{k}-G_{k}^{n}\right|_{X}^{2} d t>\frac{\epsilon^{2} \rho^{2}}{4 T^{2}}\right)+\mathbb{P}\left(\sup _{t \in[0, T]}\left|W_{k}-W_{k}^{n}\right|^{2} \int_{0}^{T}\left|G_{k}\right|_{X}^{2} d t>\frac{\epsilon^{2} \rho^{2}}{4 T^{2}}\right)
\end{aligned}
$$

The final term in (8.4) yields to an identical estimate.

Collecting the estimates $(8.5),(8.6),(8.7),(8.8)$ we infer that

$$
\begin{aligned}
& \mathbb{P}\left(\left|Y_{k}^{n}-Y_{k}\right|_{L^{2}([0, T], X)}>5 \epsilon\right) \\
& \leq 2 \delta+2 \mathbb{P}\left(\int_{0}^{T}\left|G_{k}^{n}-G_{k}\right|_{X}^{2} d t>\frac{\delta \epsilon^{2}}{3 T}\right)+2 \mathbb{P}\left(\int_{0}^{T}\left|G_{k}-\mathcal{R}_{\rho}\left(G_{k}\right)\right|_{X}^{2} d t>\frac{\delta \epsilon^{2}}{3 T}\right) \\
& \quad+3 \mathbb{P}\left(\sup _{t \in[0, T]}\left|W_{k}^{n}\right|^{2} \int_{0}^{T}\left|G_{k}-G_{k}^{n}\right|_{X}^{2} d t>\frac{\epsilon^{2} \rho^{2}}{4 T}\right)+3 \mathbb{P}\left(\sup _{t \in[0, T]}\left|W_{k}-W_{k}^{n}\right|^{2} \int_{0}^{T}\left|G_{k}\right|_{X}^{2} d t>\frac{\epsilon^{2} \rho^{2}}{4 T}\right)
\end{aligned}
$$

Since $\delta, \epsilon>0$ are arbitrary and given basic properties of the functional (8.3) along with (2.14) we may now infer the second item of (8.1) from (8.9). This completes the proof of Lemma 2.1.

\section{Acknowledgments}

This work was partially supported by the National Science Foundation under the grants NSF grants DMS1004638, DMS-0906440, and by the Research Fund of Indiana University.

\section{References}

[1] A. Bensoussan. Stochastic Navier-Stokes equations. Acta Appl. Math., 38(3):267-304, 1995.

[2] A. Bensoussan and J. Frehse. Local solutions for stochastic Navier Stokes equations. M2AN Math. Model. Numer. Anal., 34(2):241-273, 2000. Special issue for R. Temam's 60th birthday.

[3] A. Bensoussan and R. Temam. Équations stochastiques du type Navier-Stokes. J. Functional Analysis, 13:195-222, 1973.

[4] J. Berner, G. J. Shutts, M. Leutbecher, and T. N. Palmer. A spectral stochastic kinetic energy backscatter scheme and its impact on flow-dependent predictability in the ecmwf ensemble prediction system. Journal of the Atmospheric Sciences, 66(3):603-626, 2009.

[5] H. Breckner. Galerkin approximation and the strong solution of the Navier-Stokes equation. J. Appl. Math. Stochastic Anal., 13(3):239-259, 2000.

[6] Z. Brzeźniak and S. Peszat. Strong local and global solutions for stochastic Navier-Stokes equations. In Infinite dimensional stochastic analysis (Amsterdam, 1999), volume 52 of Verh. Afd. Natuurkd. 1. Reeks. K. Ned. Akad. Wet., pages 85-98. R. Neth. Acad. Arts Sci., Amsterdam, 2000.

[7] C. Cao and E. Titi. Global well-posedness of the three-dimensional viscous primitive equations of large scale ocean and atmosphere dynamics. Ann. of Math. (2), 166(1):245-267, 2007. 
[8] M. Capiński and D. Gatarek. Stochastic equations in Hilbert space with application to Navier-Stokes equations in any dimension. J. Funct. Anal., 126(1):26-35, 1994.

[9] A. B. Cruzeiro. Solutions et mesures invariantes pour des équations d'évolution stochastiques du type Navier-Stokes. Exposition. Math., 7(1):73-82, 1989.

[10] G. Da Prato and A. Debussche. Ergodicity for the 3D stochastic Navier-Stokes equations. J. Math. Pures Appl. (9), 82(8):877-947, 2003.

[11] G. Da Prato and J. Zabczyk. Stochastic equations in infinite dimensions, volume 44 of Encyclopedia of Mathematics and its Applications. Cambridge University Press, Cambridge, 1992.

[12] G. Da Prato and J. Zabczyk. Ergodicity for infinite-dimensional systems, volume 229 of London Mathematical Society Lecture Note Series. Cambridge University Press, Cambridge, 1996.

[13] A. Debussche, N. Glatt-Holtz, R. Temam, and M. Ziane. Global existence and regularity for the 3d stochastic primitive equations of the ocean and atmosphere with multiplicative white noise. (in preparation).

[14] B. Ewald and C. Penland. Numerical generation of stochastic differential equations in climate models. In Special Volume on Computational Methods for the Atmosphere and the Oceans, volume 14 of Handbook of Numerical Analysis, pages 279-306. Elsevier/North-Holland, Amsterdam, 2009.

[15] B. Ewald, M. Petcu, and R. Temam. Stochastic solutions of the two-dimensional primitive equations of the ocean and atmosphere with an additive noise. Anal. Appl. (Singap.), 5(2):183-198, 2007.

[16] F. Flandoli. An introduction to 3d stochastic fluid dynamics. In SPDE in Hydrodynamic: Recent Progress and Prospects, volume 1942 of Lecture Notes in Mathematics, pages 51-150. Springer Berlin / Heidelberg, 2008.

[17] F. Flandoli and D. Gatarek. Martingale and stationary solutions for stochastic Navier-Stokes equations. Probab. Theory Related Fields, 102(3):367-391, 1995.

[18] F. Flandoli and M. Romito. Markov selections for the 3D stochastic Navier-Stokes equations. Probab. Theory Related Fields, 140(3-4):407-458, 2008.

[19] Franco Flandoli and Marco Romito. Partial regularity for the stochastic Navier-Stokes equations. Trans. Amer. Math. Soc., 354(6):2207-2241 (electronic), 2002.

[20] G. B. Folland. Real analysis. Pure and Applied Mathematics (New York). John Wiley \& Sons Inc., New York, second edition, 1999. Modern techniques and their applications, A Wiley-Interscience Publication.

[21] Ч. İ. Gīhman and A. V. Skorohod. Stochastic differential equations. Springer-Verlag, New York, 1972. Translated from the Russian by Kenneth Wickwire, Ergebnisse der Mathematik und ihrer Grenzgebiete, Band 72.

[22] N. Glatt-Holtz. Well Posedness and Asymptotic Analysis for the Stochastic Equations of Geophysical Fluid Dynamics. PhD thesis, University of Southern California, May 2008.

[23] N. Glatt-Holtz and Ziane M. Strong pathwise solutions of the stochastic Navier-Stokes system. Advances in Differential Equations, 14(5-6):567-600, 2009.

[24] N. Glatt-Holtz and R. Temam. Cauchy convergence schemes for some nonlinear partial differential equations. Applicable Analysis. (to appear).

[25] N. Glatt-Holtz and R. Temam. Pathwise solutions of the 2-d stochastic primitive equations. Applied Mathematics and Optimization. (to appear).

[26] N. Glatt-Holtz and M. Ziane. The stochastic primitive equations in two space dimensions with multiplicative noise. Discrete Contin. Dyn. Syst. Ser. B, 10(4):801-822, 2008.

[27] B. Guo and D. Huang. 3d stochastic primitive equations of the large-scale ocean: global well-posedness and attractors. Commun. Math. Phys., 286:697-723, 2009.

[28] I. Gyöngy and N. Krylov. Existence of strong solutions for Itô's stochastic equations via approximations. Probab. Theory Related Fields, 105(2):143-158, 1996.

[29] J. Jacod. Calcul stochastique et problèmes de martingales, volume 714 of Lecture Notes in Mathematics. Springer, Berlin, 1979.

[30] G. M. Kobelkov. Existence of a solution 'in the large' for the 3D large-scale ocean dynamics equations. C. R. Math. Acad. Sci. Paris, 343(4):283-286, 2006.

[31] I. Kukavica and M. Ziane. On the regularity of the primitive equations of the ocean. Nonlinearity, 20(12):2739-2753, 2007.

[32] D. C. Leslie and Quarini G. L. The application of turbulence theory to the formulation of subgrid modelling procedures. Journal of Fluid Mechanics, 91:65-91, 1979.

[33] J.-L. Lions, R. Temam, and S. H. Wang. New formulations of the primitive equations of atmosphere and applications. Nonlinearity, 5(2):237-288, 1992.

[34] P. J. Mason and D. J. Thomson. Stochastic backscatter in large-eddy simulations of boundary layers. Journal of Fluid Mechanics, 242(-1):51-78, 1992. 
[35] J.-L. Menaldi and S. S. Sritharan. Stochastic 2-D Navier-Stokes equation. Appl. Math. Optim., 46(1):31-53, 2002.

[36] R. Mikulevicius and B. L. Rozovskii. Stochastic Navier-Stokes equations for turbulent flows. SIAM J. Math. Anal., 35(5):1250-1310, 2004.

[37] R. Mikulevicius and B. L. Rozovskii. Global $L_{2}$-solutions of stochastic Navier-Stokes equations. Ann. Probab., 33(1):137$176,2005$.

[38] B. Øksendal. Stochastic differential equations. Universitext. Springer-Verlag, Berlin, sixth edition, 2003. An introduction with applications.

[39] J. Pedlosky. Geophysical Fluid Dynamics. Springer Verlag, 1982.

[40] C. Penland and P. D. Sardeshmukh. The optimal growth of tropical sea surface temperature anomalies. Journal of climate, 8(8):1999-2024, 1995.

[41] Cécile Penland and Brian D. Ewald. On modelling physical systems with stochastic models: diffusion versus Lévy processes. Philos. Trans. R. Soc. Lond. Ser. A Math. Phys. Eng. Sci., 366(1875):2457-2476, 2008.

[42] M. Petcu, R. Temam, and M. Ziane. Some mathematical problems in geophysical fluid dynamics. In Special Volume on Computational Methods for the Atmosphere and the Oceans, volume 14 of Handbook of Numerical Analysis, pages 577-750. Elsevier, 2008.

[43] C. Prévôt and M. Röckner. A concise course on stochastic partial differential equations, volume 1905 of Lecture Notes in Mathematics. Springer, Berlin, 2007.

[44] H. A. Rose. Eddy diffusivity, eddy noise and subgrid-scale modelling. Journal of Fluid Mechanics, 81:719-734, 1977.

[45] Armen Shirikyan. Qualitative properties of stationary measures for three-dimensional Navier-Stokes equations. J. Funct. Anal., 249(2):284-306, 2007.

[46] R. Temam. Navier-Stokes equations and nonlinear functional analysis, volume 66 of CBMS-NSF Regional Conference Series in Applied Mathematics. Society for Industrial and Applied Mathematics (SIAM), Philadelphia, PA, second edition, 1995.

[47] R. Temam. Navier-Stokes equations: Theory and numerical analysis. AMS Chelsea Publishing, Providence, RI, 2001. Reprint of the 1984 edition.

[48] M. Viot. Solutions faibles d'équations aux dérivées partielles non linéaires. 1976. Thèse, Université Pierre et Marie Curie, Paris.

[49] M. Ziane. Regularity results for Stokes type systems. Appl. Anal., 58(3-4):263-292, 1995.

[50] M. J. Zidikheri and J. S. Frederiksen. Stochastic subgrid-scale modelling for non-equilibrium geophysical flows. Philosophical Transactions of the Royal Society A: Mathematical, Physical and Engineering Sciences, 368(1910):145-160, 2010.

Arnaud Debussche

Département de Mathématiques

ENS Cachan Bretagne

Web: http://w3.bretagne.ens-cachan.fr/math/people/arnaud.debussche/

Email: arnaud.debussche@bretagne.ens-cachan.fr

Nathan Glatt-Holtz

Department of Mathematics

Indiana University

Web: http://mypage.iu.edu/ negh/

Email: negh@indiana.edu

Roger Temam

Department of Mathematics

Indiana University

Web: http://mypage.iu.edu/ temam/

Email: temam@indiana.edu 